\title{
Pacific
}

Journal of

Mathematics

\section{EXHAUSTING CURVE COMPLEXES BY FINITE RIGID SETS}

\section{JAVIER ARAMAYONA AND CHRISTOPHER J. LEININGER}




\title{
EXHAUSTING CURVE COMPLEXES BY FINITE RIGID SETS
}

\author{
JAVIER ARAMAYONA AND CHRISTOPHER J. LEININGER
}

\begin{abstract}
Let $S$ be a connected orientable surface of finite topological type. We prove that there is an exhaustion of the curve complex $\mathscr{C}(S)$ by a sequence of finite rigid sets.
\end{abstract}

\section{Introduction}

The curve complex $\mathscr{C}(S)$ of a surface $S$ is a simplicial complex whose $k$-simplices correspond to sets of $k+1$ distinct isotopy classes of essential simple closed curves on $S$ with pairwise disjoint representatives. The extended mapping class group $\operatorname{Mod}^{ \pm}(S)$ of $S$ acts on $\mathscr{C}(S)$ by simplicial automorphisms, and a well-known theorem due to Ivanov [1997], Korkmaz [1999] and Luo [2000] asserts that $\mathscr{C}(S)$ is simplicially rigid for $S \neq S_{1,2}$. More concretely, the natural homomorphism

$$
\operatorname{Mod}^{ \pm}(S) \rightarrow \operatorname{Aut}(\mathscr{C}(S))
$$

is surjective unless $S=S_{1,2}$; in the case $S=S_{1,2}$ there is an automorphism of $\mathscr{C}(S)$ that sends a separating curve on $S$ to a nonseparating one and thus cannot be induced by an element of $\operatorname{Mod}^{ \pm}(S)$ (see [Luo 2000]).

In [Aramayona and Leininger 2013], henceforth abbreviated [AL], we extended this picture and showed that curve complexes are finitely rigid. Specifically, for $S \neq S_{1,2}$ we identified a finite subcomplex $\mathfrak{X}(S) \subset \mathscr{C}(S)$ with the property that every locally injective map $\mathfrak{X}(S) \rightarrow \mathscr{C}(S)$ is the restriction of an element of $\operatorname{Mod}^{ \pm}(S)$; in the case of $S_{1,2}$ a similar statement can be made, this time using the group $\operatorname{Aut}(\mathscr{C}(S))$ instead of $\operatorname{Mod}^{ \pm}(S)$. We refer to such a subset $\mathfrak{X}(S)$ as a rigid set.

The rigid sets constructed in [AL] enjoy some curious properties. For instance, if $S=S_{0, n}$ is a sphere with $n$ punctures then $\mathfrak{X}(S)$ is homeomorphic to an $(n-4)$ dimensional sphere. Since $\mathscr{C}(S)$ has dimension $n-4$, it follows that $\mathfrak{X}(S)$ represents a nontrivial element of $H_{n-4}(\mathscr{C}(S), \mathbb{Z})$ which, by a result of Harer [1986], is the only nontrivial homology group of $\mathscr{C}(S)$. In fact, Broaddus [2012] and Birman, Broaddus and Menasco [Birman et al. 2015] have recently proved that $\mathfrak{X}(S)$ is a

Aramayona was supported by BQR (Toulouse) and Campus Iberus grants. Leininger was supported by NSF grants DMS 0905748 and 1207183.

MSC2010: 57M99.

Keywords: curve complex, mapping class group, rigidity. 
generator of $H_{n-4}(\mathscr{C}(S), \mathbb{Z})$ when viewed as a $\operatorname{Mod}^{ \pm}(S)$-module; in the case when $S$ has genus $\geq 2$ and at least one puncture, they prove that $\mathfrak{X}(S)$ contains a generator for the homology of $\mathscr{C}(S)$.

The rigid sets identified in [AL] all have diameter 2 in $\mathscr{C}(S)$, and a natural question is whether there exist finite rigid sets in $\mathscr{C}(S)$ of arbitrarily large diameter; see Question 1 of that work. In this paper we prove that, in fact, there exists an exhaustion of $\mathscr{C}(S)$ by finite rigid sets:

Theorem 1.1. Let $S \neq S_{1,2}$ be a connected orientable surface of finite topological type. There exists a sequence $\mathfrak{X}_{1} \subset \mathfrak{X}_{2} \subset \cdots \subset \mathscr{C}(S)$ such that

(1) $\mathfrak{X}_{i}$ is a finite rigid set for all $i \geq 1$,

(2) $\mathfrak{X}_{i}$ has trivial pointwise stabilizer in $\operatorname{Mod}^{ \pm}(S)$, for all $i \geq 1$, and

(3) $\bigcup_{i \geq 1} \mathfrak{X}_{i}=\mathscr{C}(S)$.

Remarks. (i) A similar statement can be made for $S=S_{1,2}$, by replacing $\operatorname{Mod}^{ \pm}(S)$ by $\operatorname{Aut}(\mathscr{C}(S))$ in the definition of rigid set above.

(ii) We stress that Theorem 1.1 above does not follow from the main result in [AL]. Indeed, a subset of $\mathscr{C}(S)$ containing a rigid set need not itself be rigid; compare with Proposition 3.2 below.

(iii) The combination of a recent theorem of J. Hernández (as yet unpublished) and the main result in [AL] gives an alternate proof of Theorem 1.1 in the case when $S$ has genus $\geq 3$; compare with the remark on page 262 below.

As a consequence of Theorem 1.1 we will obtain a "finitistic" proof of the aforementioned result of [Ivanov 1997; Korkmaz 1999; Luo 2000] on the simplicial rigidity of the curve complex. In fact, we will deduce the following stronger form due to Shackleton [2007].

Corollary 1.2. Let $S \neq S_{1,2}$ be a connected orientable surface of finite topological type. If $\phi: \mathscr{C}(S) \rightarrow \mathscr{C}(S)$ is a locally injective simplicial map, then there exists $h \in \operatorname{Mod}^{ \pm}(S)$ such that $h=\phi$.

The first author and Souto [2013] proved that if $\mathfrak{X} \subset \mathscr{C}(S)$ is a rigid set satisfying some extra conditions, then every (weakly) injective homomorphism from the rightangled Artin group $\mathbb{A}(\mathfrak{X})$ into $\operatorname{Mod}^{ \pm}(S)$ is obtained, up to conjugation, by taking powers of roots of Dehn twists in the vertices of $\mathfrak{X}$. Since the finite rigid sets $\mathfrak{X}_{i}$ of Theorem 1.1 all satisfy the conditions of [Aramayona and Souto 2013], we obtain the following result; here, $T_{\gamma}$ denotes the Dehn twist about $\gamma$.

Corollary 1.3. Let $S \neq S_{1,2}$ be a connected orientable surface of finite topological type, and consider the sequence $\mathfrak{X}_{1} \subset \mathfrak{X}_{2} \subset \cdots \subset \mathscr{C}(S)$ of finite rigid sets given by 
Theorem 1.1. If $\rho_{i}: \mathbb{A}\left(\mathfrak{X}_{i}\right) \rightarrow \operatorname{Mod}^{ \pm}(S)$ is an injective homomorphism, then there exist functions $a, b: \mathscr{C}^{(0)}(S) \rightarrow \mathbb{Z} \backslash\{0\}$ and $f_{i} \in \operatorname{Mod}^{ \pm}(S)$ such that

$$
\rho_{i}\left(\gamma^{a(\gamma)}\right)=f_{i} T_{\gamma}^{b(\gamma)} f_{i}^{-1}
$$

for every vertex $\gamma$ of $\mathfrak{X}_{i}$.

We remark that Kim and Koberda [2013] have previously shown the existence of injective homomorphisms $\mathbb{A}\left(Y_{i}\right) \rightarrow \operatorname{Mod}^{ \pm}(S)$ for sequences $Y_{1} \subset Y_{2} \subset \cdots$ of subsets of $\mathscr{C}(S)$. Such homomorphisms may in fact be obtained by sending a generator of $\mathbb{A}\left(Y_{i}\right)$ to a sufficiently high power of a Dehn multitwist, see [Kim and Koberda 2015].

Plan of the paper. In Section 2 we recall some necessary definitions and basic results from our previous paper [AL]. Section 3 deals with the problem of enlarging a rigid set in such a way that it remains rigid. As was the case in [AL], the techniques used in the proof of our main result differ depending on the genus of $S$. As a result, we prove Theorem 1.1 for surfaces of genus $g=0, g \geq 2$ and $g=1$ in Sections 4, 5 and 6 , respectively.

\section{Definitions}

Let $S=S_{g, n}$ be an orientable surface of genus $g$ with $n$ punctures and/or marked points. We define the complexity of $S$ as $\xi(S)=3 g-3+n$. We say that a simple closed curve on $S$ is essential if it does not bound a disk or a once-punctured disk on $S$. An essential subsurface of $S$ is a properly embedded subsurface $N \subset S$ for which each boundary component is an essential curve in $S$.

The curve complex $\mathscr{b}(S)$ of $S$ is a simplicial complex whose $k$-simplices correspond to sets of $k+1$ isotopy classes of essential simple closed curves on $S$ with pairwise disjoint representatives. In order to simplify the notation, a set of isotopy classes of simple closed curves will be confused with its representative curves, the corresponding vertices of $\mathscr{C}(S)$, and the subcomplex of $\mathscr{C}(S)$ spanned by the vertices. We also assume that representatives of isotopy classes of curves and subsurfaces intersect minimally (that is, transversely and in the minimal number of components), and denote by $i(\alpha, \beta)$ their intersection number.

If $\xi(S)>1$, then $\mathscr{C}(S)$ is a connected complex of dimension $\xi(S)-1$. If $\xi(S) \leq 0$ and $S \neq S_{1,0}$, then $\mathscr{C}(S)$ is empty. If $\xi(S)=1$ or $S=S_{1,0}$, then $\mathscr{C}(S)$ is a countable set of vertices; in order to obtain a connected complex, we modify the definition of $\mathscr{C}(S)$ by declaring $\alpha, \beta \in \mathscr{C}^{(0)}(S)$ to be adjacent in $\mathscr{C}(S)$ whenever $i(\alpha, \beta)=1$ if $S=S_{1,1}$ or $S=S_{1,0}$, and whenever $i(\alpha, \beta)=2$ if $S=S_{0,4}$. Furthermore, we add triangles to make $\mathscr{C}(S)$ into a flag complex. In all three cases, the complex $\mathscr{C}(S)$ so obtained is isomorphic to the well-known Farey complex.

We recall some definitions and results from $[\mathrm{AL}]$ that we will need later. 
Definition 2.1 (detectable intersection). Let $S$ be a surface and $Y \subset \mathscr{C}(S)$ a subcomplex. If $\alpha, \beta \in Y$ are curves with $i(\alpha, \beta) \neq 0$, then we say that their intersection is $Y$-detectable (or simply detectable if $Y$ is understood) if there are two pants decompositions $P_{\alpha}, P_{\beta} \subset Y$ such that

$$
\alpha \in P_{\alpha}, \quad \beta \in P_{\beta} \quad \text { and } \quad P_{\alpha}-\alpha=P_{\beta}-\beta .
$$

We note that if $\alpha, \beta$ have detectable intersection, then they must fill a $\xi=1$ (essential) subsurface, which we denote $N(\alpha \cup \beta) \subset S$. For notational purposes, we call $P=P_{\alpha}-\alpha=P_{\beta}-\beta$ a pants decomposition of $S-N(\alpha \cup \beta)$, even though it includes the boundary components of $N(\alpha \cup \beta)$. The following lemma is Lemma 2.3 in $[\mathrm{AL}]$.

Lemma 2.2. Let $Y \subset \mathscr{C}(S)$ be a subcomplex, and $\alpha, \beta \in Y$ intersecting curves with $Y$-detectable intersection. If $\phi: Y \rightarrow \mathscr{C}(S)$ is a locally injective simplicial map, then $\phi(\alpha), \phi(\beta)$ have $\phi(Y)$-detectable intersection, and hence fill a $\xi=1$ subsurface.

Farey neighbors. A large part of our arguments will rely on being able to recognize when two curves are Farey neighbors, which we now define.

Definition 2.3 (Farey neighbors). Let $\alpha$ and $\beta$ be curves on $S$ which fill a $\xi=1$ subsurface $N \subset S$. We say $\alpha$ and $\beta$ are Farey neighbors if they are adjacent in $\mathscr{C}(N)$.

The following result is a useful tool for recognizing Farey neighbors, and is a rephrasing of Lemma 2.4 in [AL] (see also the comment immediately after it):

Lemma 2.4. Suppose $\alpha_{1}, \alpha_{2}, \alpha_{3}, \alpha_{4}$ are curves on $S$ such that

(1) $\alpha_{2}, \alpha_{3}$ together fill a $\xi=1$ subsurface $N \subset S$,

(2) $i\left(\alpha_{i}, \alpha_{j}\right)=0 \Leftrightarrow|i-j|>1$ for all $i \neq j$, and

(3) $\alpha_{1}$ and $\alpha_{4}$ have nonzero intersection number with exactly one component of $\partial N$.

Then $\alpha_{2}$ and $\alpha_{3}$ are Farey neighbors.

\section{Enlarging rigid sets}

In this section we discuss the problem of enlarging rigid sets of the curve complex. We recall the definition of rigid set from [AL].

Definition 3.1 (rigid set). Suppose $S \neq S_{1,2}$. We say that $Y \subset \mathscr{C}(S)$ is rigid if for every locally injective simplicial map $\phi: Y \rightarrow \mathscr{C}(S)$ there exists $h \in \operatorname{Mod}^{ \pm}(S)$ with $\left.h\right|_{Y}=\phi$, unique up to the pointwise stabilizer of $Y$ in $\operatorname{Mod}^{ \pm}(S)$.

Remark. The definition above may seem somewhat different from the one used in [AL], where we used the group $\operatorname{Aut}(\mathscr{C}(S))$ instead of $\operatorname{Mod}^{ \pm}(S)$. Nevertheless, in light of the results of Ivanov [1997], Korkmaz [1999] and Luo [2000] mentioned in the introduction, the two definitions are essentially the same as $S \neq S_{1,2}$. For 
$S=S_{1,2}$, however, we will use the group $\operatorname{Aut}(\mathscr{C}(S))$ instead of $\operatorname{Mod}^{ \pm}(S)$, due to the existence of nongeometric automorphisms of $\mathscr{C}(S)$.

The main step in the proof of Theorem 1.1 is to enlarge the rigid sets constructed in $[\mathrm{AL}]$ in a way that the sets we obtain remain rigid. As we mentioned in the introduction, while one might be tempted to guess that a set that contains a rigid set is necessarily rigid, this is far from true, as the next result shows.

Proposition 3.2. Let $S=S_{0, n}$, with $n \geq 5$, and $\mathfrak{X}$ the finite rigid set identified in $[A L]$ (defined in Section 4). For every curve $\alpha \in \mathscr{C}(S) \backslash \mathfrak{X}$, the set $\mathfrak{X} \cup\{\alpha\}$ is not rigid.

Proof. Let $S_{\alpha}$ be the smallest subsurface of $S$ containing all the curves in $\mathfrak{X}$ which are disjoint from $\alpha$. Observe that since $\mathfrak{X}$ is rigid, it is also filling and therefore $S_{\alpha}$ is a proper subsurface of $S$. Let $S_{\alpha}^{\prime}$ be the connected component of $S \backslash S_{\alpha}$ that contains $\alpha$; from the construction in [AL], every component of $\partial S_{\alpha}^{\prime}$ which is essential in $S$ is an element of $\mathfrak{X}$. We claim that there exists $f \in \operatorname{Mod}(S)$ with the following two properties:

(1) The restriction of $f$ to $S_{\alpha}$ is the identity map.

(2) For every $\beta \in \mathfrak{X}$ with $i(\alpha, \beta) \neq 0$, we have $i(f(\alpha), \beta) \neq 0$.

In order to construct such an $f$, one can for instance consider an element $h \in \operatorname{Mod}(S)$ that is pseudo-Anosov on $S_{\alpha}^{\prime}$ and the identity on $S_{\alpha}$; any sufficiently high power of $h$ will satisfy the two conditions above.

At this point, define a map $\phi: \mathfrak{X} \cup\{\alpha\} \rightarrow \mathscr{C}(S)$ by $\phi(\beta)=\beta$ for all $\beta \neq \alpha$, and $\phi(\alpha)=f(\alpha)$. By construction, the map $\phi$ is locally injective and simplicial, but cannot be the restriction of an element of $\operatorname{Mod}^{ \pm}(S)$.

While Proposition 3.2 serves to highlight the obstacles for enlarging a rigid set to a set that is also rigid, we now explain two procedures for doing so. First, we recall the following definition from [AL].

Definition 3.3. Let $A$ be a set of curves in $S$.

(1) $A$ is almost filling (in $S$ ) if the set

$$
B=\left\{\beta \in \mathscr{C}^{(0)}(S) \backslash A \mid i(\alpha, \beta)=0 \forall \alpha \in A\right\}
$$

is finite. In this case, we call $B$ the set of curves determined by $A$.

(2) If $A$ is almost filling (in $S$ ), and $B=\{\beta\}$ is a single curve, then we say that $\beta$ is uniquely determined by $A$.

An immediate consequence of the definition is the following.

Lemma 3.4. Let $Y$ be a rigid set of curves, and $A \subset Y$ an almost filling set in $S$. If $\beta$ is uniquely determined by $A$, then $Y \cup\{\beta\}$ is rigid. 
Proof. Given any locally injective simplicial map $\phi: Y \cup\{\beta\} \rightarrow \mathscr{C}(S)$, we let $f \in \operatorname{Mod}^{ \pm}(\mathscr{C}(S))$ be such that $\left.f\right|_{Y}=\phi$. Then $f(\beta)$ is the unique curve determined by $f(A)=\phi(A)$. On the other hand, $\phi(\beta)$ is connected by an edge to every vertex in $\phi(A)$, since $\phi$ is simplicial. Since $\phi$ is injective on the star of $\beta$, it is injective on $\beta \cup A$, and so $\phi(\beta) \notin A$. It follows that $\phi(\beta)$ is the curve uniquely determined by $\phi(A)$, and hence $f(\beta)=\phi(\beta)$.

In particular, this gives rise to one method for enlarging a rigid set which we formalize as follows. Given a subset $Y \subset \mathscr{C}(S)$, define

$Y^{\prime}=Y \cup\{\beta \mid \beta$ is uniquely determined by some almost filling set $A \subset Y\}$.

From this we recursively define $Y=Y^{0}$ and $Y^{r}=\left(Y^{r-1}\right)^{\prime}$ for all $r>0$. Observe that, as an immediate consequence of Lemma 3.4, we obtain:

Proposition 3.5. If $Y \subset \mathscr{C}(S)$ is a rigid set, then so is $Y^{r}$ for all $r \geq 0$.

Remark. J. Hernández has recently proved that for every surface $S$ of genus $\geq 3$, there exists an explicit finite subcomplex $Y \subset \mathscr{C}(S)$ such that

$$
\bigcup_{r \geq 0} Y^{r}=\mathscr{C}(S) \text {. }
$$

As a corollary of this result, and using the main result in [AL], he provides an alternate proof of Theorem 1.1; compare with remark (iii) on page 258 above.

Next, we give a sufficient condition for the union of two rigid sets to be rigid. Before doing so, we need the following definition.

Definition 3.6 (weakly rigid set). We say that a set $Y \subset \mathscr{C}(S)$ is weakly rigid if, whenever $h, h^{\prime} \in \operatorname{Mod}^{ \pm}(S)$ satisfy $\left.h\right|_{Y}=\left.h^{\prime}\right|_{Y}$, then $h=h^{\prime}$.

Alternatively, $Y$ is weakly rigid if the pointwise stabilizer in $\operatorname{Mod}^{ \pm}(S)$ is trivial. Note that if $Y$ is a weakly rigid set, then so is every set containing $Y$.

Lemma 3.7. Let $Y_{1}, Y_{2} \subset \mathscr{C}(S)$ be rigid sets. If $Y_{1} \cap Y_{2}$ is weakly rigid then $Y_{1} \cup Y_{2}$ is rigid.

Proof. Let $\phi: Y_{1} \cup Y_{2} \rightarrow \mathscr{C}(S)$ be a locally injective simplicial map. Since $Y_{i}$ is rigid and has trivial pointwise stabilizer in $\operatorname{Mod}^{ \pm}(S)$ (because $Y_{1} \cap Y_{2}$ does), there exists a unique $h_{i} \in \operatorname{Mod}^{ \pm}(S)$ such that $\left.h_{i}\right|_{Y_{i}}=\left.\phi\right|_{Y_{i}}$. Finally, since $Y_{1} \cap Y_{2}$ is weakly rigid we have $h_{1}=h_{2}=h$. Therefore $\left.h\right|_{Y_{1} \cup Y_{2}}=\phi$, and the result follows.

We now proceed to describe our second method for enlarging a rigid set. We start with some definitions and notation. We write $T_{\alpha}$ for the Dehn twist along a curve $\alpha$. Recall that the half-twist $H_{\alpha}$ about a curve $\alpha$ is defined if and only if the curve cuts off a pair of pants containing two punctures of $S$. Furthermore, there is exactly one half-twist about $\alpha$ if in addition $S$ is not a four-holed sphere. 
Definition 3.8. Farey neighbors $\alpha$ and $\beta$ are twistable if either

(1) $N(\alpha \cup \beta)$ is a one-holed torus, or

(2) $N(\alpha \cup \beta)$ is a four-holed sphere and $H_{\alpha}, H_{\beta}$ are both defined and unique.

In this situation we define $f_{\alpha}=T_{\alpha}$ and $f_{\beta}=T_{\beta}$ in the first case and $f_{\alpha}=H_{\alpha}$ and $f_{\beta}=H_{\beta}$ in the second. We call $f_{\alpha}, f_{\beta}$ the twisting pair for $\alpha, \beta$.

In case (1) we call $\alpha$ and $\beta$ toroidal and in case (2) we call them spherical.

We note that whether twistable Farey neighbors $\alpha$ and $\beta$ are toroidal or spherical can be distinguished

(i) by $i(\alpha, \beta)$ (whether it is 1 or 2$)$,

(ii) by the homeomorphism types of $\alpha$ and $\beta$ (whether they are nonseparating curves or they cut off a pair of pants), or

(iii) by the homeomorphism type of $N(\alpha \cup \beta)$ (whether it is a one-holed torus or a four-holed sphere).

The following well-known fact describes the common feature of these two situations.

Proposition 3.9. Suppose $\alpha, \beta$ are twistable Farey neighbors and that $f_{\alpha}, f_{\beta}$ is their twisting pair. Then

$$
f_{\alpha}(\beta)=f_{\beta}^{-1}(\alpha) \text { and } f_{\alpha}^{-1}(\beta)=f_{\beta}(\alpha),
$$

and these are the unique common Farey neighbors of both $\alpha$ and $\beta$.

Sets of twistable Farey neighbors which interact with each other frequently occur in our rigid sets. We distinguish one particular type of such sets in the following definition.

Definition 3.10. Suppose $Y$ is a rigid subset of $\mathscr{C}(S)$ and $A=\left\{\alpha_{1}, \ldots, \alpha_{k}\right\} \subset Y$. We say that $A$ is a closed string of Farey neighbors in $Y$ provided the following conditions are satisfied, counting indices modulo $k$ :

(1) The curves $\alpha_{i}, \alpha_{i+1}$ are twistable Farey neighbors with twisting pair $f_{\alpha_{i}}, f_{\alpha_{i+1}}$.

(2) $i\left(\alpha_{i}, \alpha_{i+1}\right) \neq 0$ is $Y$-detectable.

(3) $i\left(\alpha_{i}, \alpha_{j}\right)=0$ if $i-j \neq \pm 1$ modulo $k$.

(4) $\alpha_{i}, \alpha_{i+1}, \alpha_{i+2}, \alpha_{i+3}$ satisfy the hypothesis of Lemma 2.4.

Given a closed string of twistable Farey neighbors $A \subset Y$, we define

$$
Y_{A}=Y \cup\left\{f_{\alpha_{i}}^{ \pm 1}\left(\alpha_{j}\right)\right\}_{i, j=1}^{k} .
$$

Remark. Two comments are in order: 
(1) There is a priori some ambiguity in the notation as $f_{\alpha_{i}}$ can be defined as part of the twisting pair for $\alpha_{i}, \alpha_{i+1}$ as well as for $\alpha_{i-1}, \alpha_{i}$. However, if $\alpha_{i}$ is part of two pairs of different twistable Farey neighbors in $Y$, then they must both be toroidal or both spherical as this is determined by the homeomorphism type of $\alpha_{i}$. Consequently, the mapping class $f_{\alpha_{i}}$ is independent of what twistable pair it is included in.

(2) Given condition (3) of Definition 3.10, the set $Y_{A}$ has a more descriptive definition. Namely,

$$
Y_{A}=Y \cup\left\{f_{\alpha_{i}}^{ \pm 1}\left(\alpha_{j}\right) \mid i-j= \pm 1 \text { modulo } k\right\} .
$$

See Figure 1 for an example of a closed string of twistable Farey neighbors and two of their images under the twisting pair.

The situation in the next proposition arises in multiple settings, and provides a way to extend a rigid set to a larger set which is nearly rigid.

Proposition 3.11. Let $Y$ be a rigid subset of $\mathscr{b}(S)$ and $A=\left\{\alpha_{1}, \ldots, \alpha_{k}\right\} \subset Y$ a closed string of twistable Farey neighbors in $Y$. Then, counting indices modulo $k$ :

(1) $f_{\alpha_{i}}^{ \pm 1}\left(\alpha_{i+1}\right)=f_{\alpha_{i+1}}^{\mp 1}\left(\alpha_{i}\right)$ are the unique common Farey neighbors of $\alpha_{i}$ and $\alpha_{i+1}$.

(2) $i\left(f_{\alpha_{i}}^{ \pm 1}\left(\alpha_{j}\right), \alpha_{j^{\prime}}\right) \neq 0$ for all $i$ and all

$$
\left(j, j^{\prime}\right) \in\{(i+1, i),(i+1, i+1),(i-1, i),(i-1, i-1)\} .
$$

Furthermore, these intersections are $Y_{A}$-detectable.

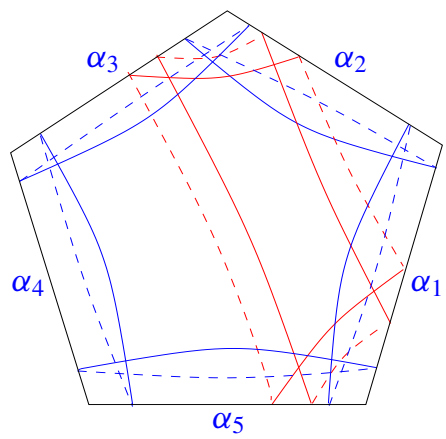

Figure 1. The set $Y=A=\left\{\alpha_{1}, \ldots, \alpha_{5}\right\}$ is the rigid set $\mathfrak{X}\left(S_{0,5}\right)$ identified in [Luo 2000] and [AL], and is a closed string of twistable Farey neighbors. The red curves in the picture are $f_{\alpha_{1}}\left(\alpha_{2}\right), f_{\alpha_{2}}\left(\alpha_{1}\right)$, for the twistable pair $\alpha_{1}, \alpha_{2}$. The automorphism group of $Y_{A}$ that fixes $Y$ pointwise is generated by an orientation-reversing involution $\sigma: S_{0,5} \rightarrow S_{0,5}$ that fixes $\alpha_{i}$ and interchanges $f_{\alpha_{i}}$ and $f_{\alpha_{i+1}}$, for all $i(\bmod 5)$. 
(3) For any locally injective simplicial map $\phi: Y_{A} \rightarrow \mathscr{C}(S)$,

$$
\phi\left(f_{\alpha_{i}}\left(\alpha_{i+1}\right)\right)=\phi\left(f_{\alpha_{i+1}}^{-1}\left(\alpha_{i}\right)\right) \quad \text { and } \quad \phi\left(f_{\alpha_{i}}^{-1}\left(\alpha_{i+1}\right)\right)=\phi\left(f_{\alpha_{i+1}}\left(\alpha_{i}\right)\right)
$$

are the unique Farey neighbors of $\phi\left(\alpha_{i}\right)$ and $\phi\left(\alpha_{i+1}\right)$.

Proof. Conclusion (1) follows immediately from Definition 3.10 part (1) and Proposition 3.9.

Next we prove conclusion (2). Fix $\left(j, j^{\prime}\right)$ as in the proposition. Then since $i\left(\alpha_{i}, \alpha_{j}\right) \neq 0$, it follows that $f_{\alpha_{i}}\left(\alpha_{j}\right)$ nontrivially intersects both $\alpha_{i}$ and $\alpha_{j}$. Since $\alpha_{j^{\prime}}$ is one of these latter two curves, the first statement follows. By part (2) of Definition 3.10, $i\left(\alpha_{i}, \alpha_{j}\right) \neq 0$ is $Y$-detectable. Let $P_{\alpha_{i}}, P_{\alpha_{j}} \subset Y$ be pants decompositions containing $\alpha_{i}$ and $\alpha_{j}$, respectively, as in Definition 2.1, and set $P=P_{\alpha_{i}}-\alpha_{i}=P_{\alpha_{j}}-\alpha_{j}$. Then since $f_{\alpha_{i}}$ is supported in $N\left(\alpha_{i} \cup \alpha_{j}\right)$ which is contained in the complement of $P$, we can define two more pants decompositions

$$
P_{f_{\alpha_{i}}^{ \pm 1}\left(\alpha_{j}\right)}=P \cup f_{\alpha_{i}}^{ \pm 1}\left(\alpha_{j}\right) \subset Y_{A} .
$$

Together with $P_{\alpha_{i}}$ and $P_{\alpha_{j}}$ these are sufficient to detect all the intersections claimed. In all cases, $P \subset Y$ is the pants decomposition of the complement of $N\left(\alpha_{i} \cup \alpha_{j}\right)$, as required.

For conclusion (3), we explain why $\phi\left(f_{\alpha_{i}}\left(\alpha_{i+1}\right)\right)$ and $\phi\left(\alpha_{i}\right)$ are Farey neighbors. The other three cases are similar. For this, we consider the set

$$
\left\{\phi\left(f_{\alpha_{i}}\left(\alpha_{i-1}\right)\right), \phi\left(\alpha_{i}\right)=\phi\left(f_{\alpha_{i}}\left(\alpha_{i}\right)\right), \phi\left(f_{\alpha_{i}}\left(\alpha_{i+1}\right)\right), \phi\left(\alpha_{i+2}\right)=\phi\left(f_{\alpha_{i}}\left(\alpha_{i+2}\right)\right)\right\} .
$$

The equalities here follow from the disjointness property (3) of Definition 3.10 since a Dehn twist or half-twist has no effect on a curve that is disjoint from the curve supporting the twist. The goal is to prove that all three conditions of Lemma 2.4 are satisfied.

By part (2) of the proposition and Lemma 2.2 it follows that any two consecutive curves in this set have $\phi\left(Y_{A}\right)$-detectable intersections and fill a $\xi=1$ subsurface. Therefore condition (1) of Lemma 2.4 is satisfied for this set of curves. Since $\alpha_{i-1}, \alpha_{i}, \alpha_{i+1}, \alpha_{i+2}$ satisfy condition (2) of Lemma 2.4 and the given set is the image of these under the simplicial map $\phi \circ f_{\alpha_{i}}$, these curves also satisfy condition (2) of Lemma 2.4.

Finally, we wish to verify that condition (3) of Lemma 2.4 is satisfied. Since $Y$ is rigid, there exists $f \in \operatorname{Mod}^{ \pm 1}(S)$ inducing $\left.\phi\right|_{Y}$. We also note that

$$
N=N\left(\alpha_{i} \cup \alpha_{i+1}\right)=N\left(\alpha_{i} \cup f_{\alpha_{i}}\left(\alpha_{i+1}\right)\right)
$$

has only one boundary component - all other holes of this subsurface (if any) must be punctures of $S$. Since the pants decomposition of the complement of $N$ is

$$
P=P_{\alpha_{i}}-\alpha_{i}=P_{\alpha_{i+1}}-\alpha_{i+1}=P_{f_{\alpha_{i}}\left(\alpha_{i+1}\right)}-f_{\alpha_{i}}\left(\alpha_{i+1}\right)
$$


and is contained in $Y$, we see that $\phi(P)=f(P)$. Because this is used in the $\phi\left(Y_{A}\right)$-detection of both $i\left(\phi\left(\alpha_{i}\right), \phi\left(\alpha_{i+1}\right)\right) \neq 0$ and $i\left(\phi\left(\alpha_{i}\right), \phi\left(f_{\alpha_{i}}\left(\alpha_{i+1}\right)\right)\right) \neq 0$, we have

$$
f(N)=N\left(f\left(\alpha_{i}\right) \cup f\left(\alpha_{i+1}\right)\right)=N\left(\phi\left(\alpha_{i}\right) \cup \phi\left(\alpha_{i+1}\right)\right)=N\left(\phi\left(\alpha_{i}\right) \cup \phi\left(f_{\alpha_{i}}\left(\alpha_{i+1}\right)\right)\right) .
$$

Consequently, this surface has only one boundary component, and so condition (3) of Lemma 2.4 is satisfied.

Proposition 3.12. If $Y$ is a rigid subset of $\mathscr{C}(S)$ and $A=\left\{\alpha_{1}, \ldots, \alpha_{k}\right\}$ is a closed string of twistable Farey neighbors in $Y$, then any locally injective simplicial map $\phi: Y_{A} \rightarrow \mathscr{C}(S)$ which is the identity on $Y$ satisfies $\phi\left(Y_{A}\right)=Y_{A}$. Furthermore, the subgroup of the automorphism group of $Y_{A}$ fixing $Y$ pointwise has order at most 2 . If this subgroup is nontrivial, then it is generated by the involution $\sigma: Y_{A} \rightarrow Y_{A}$ given by $\sigma\left(f_{\alpha_{i}}\left(\alpha_{j}\right)\right)=f_{\alpha_{i}}^{-1}\left(\alpha_{j}\right)$ for all $i, j$ (or equivalently, for all $i, j$ with $i-j= \pm 1$ (modulo k)).

Proof. Since $f_{\alpha_{i}}^{ \pm 1}\left(\alpha_{i+1}\right)$ is the unique pair of common Farey neighbors of $\alpha_{i}, \alpha_{i+1}$, and since $\phi\left(\alpha_{i}\right)=\alpha_{i}, \phi\left(\alpha_{i+1}\right)=\alpha_{i+1}$, Proposition 3.11 implies that for every $i$ and $j$ with $i-j= \pm 1$ (modulo $k$ ), we have

$$
\left\{\phi\left(f_{\alpha_{i}}\left(\alpha_{j}\right)\right), \phi\left(f_{\alpha_{i}}^{-1}\left(\alpha_{j}\right)\right)\right\}=\left\{f_{\alpha_{i}}\left(\alpha_{j}\right), f_{\alpha_{i}}^{-1}\left(\alpha_{j}\right)\right\},
$$

and so the first claim of the proposition follows.

Next we suppose $\phi$ is any automorphism of $Y$ that restricts to the identity on $Y$. We claim that if there is some $i, j$ with $i-j= \pm 1$ (modulo $k$ ) so that $\phi\left(f_{\alpha_{i}}\left(\alpha_{j}\right)\right)=f_{\alpha_{i}}^{-1}\left(\alpha_{j}\right)$, then this is true for every $i, j$ with $i-j= \pm 1$ (modulo $k$ ). To this end, suppose that $\phi\left(f_{\alpha_{i}}\left(\alpha_{i+1}\right)\right)=f_{\alpha_{i}}^{-1}\left(\alpha_{i+1}\right)$ for some index $i$ (the case $\phi\left(f_{\alpha_{i}}\left(\alpha_{i-1}\right)\right)=f_{\alpha_{i}}^{-1}\left(\alpha_{i-1}\right)$ is similar). Then note that

$$
i\left(f_{\alpha_{i}}\left(\alpha_{i-1}\right), f_{\alpha_{i}}\left(\alpha_{i+1}\right)\right)=0=i\left(f_{\alpha_{i}}^{-1}\left(\alpha_{i-1}\right), f_{\alpha_{i}}^{-1}\left(\alpha_{i+1}\right)\right)
$$

while

$$
i\left(f_{\alpha_{i}}\left(\alpha_{i-1}\right), f_{\alpha_{i}}^{-1}\left(\alpha_{i+1}\right)\right) \neq 0 \neq i\left(f_{\alpha_{i}}^{-1}\left(\alpha_{i-1}\right), f_{\alpha_{i}}\left(\alpha_{i+1}\right)\right) .
$$

Since $\phi$ is simplicial and locally injective, we must have $\phi\left(f_{\alpha_{i}}\left(\alpha_{i-1}\right)\right)=f_{\alpha_{i}}^{-1}\left(\alpha_{i-1}\right)$ and $\phi\left(f_{\alpha_{i}}^{-1}\left(\alpha_{i-1}\right)\right)=f_{\alpha_{i}}\left(\alpha_{i-1}\right)$. Consequently,

$$
\phi\left(f_{\alpha_{i-1}}\left(\alpha_{i}\right)\right)=\phi\left(f_{\alpha_{i}}^{-1}\left(\alpha_{i-1}\right)\right)=f_{\alpha_{i}}\left(\alpha_{i-1}\right)=f_{\alpha_{i-1}}^{-1}\left(\alpha_{i}\right) .
$$

Repeating this argument again, it follows that $\phi\left(f_{\alpha_{i}}\left(\alpha_{i+1}\right)\right)=f_{\alpha_{i}}^{-1}\left(\alpha_{i+1}\right)$ for all $i$, as required. Thus, in this case, $\phi$ is given by $\sigma$ as in the statement of the proposition.

If we are not in the situation of the previous paragraph, then it follows that $\phi$ is the identity, completing the proof. 
After this discussion we are in a position to explain how to obtain an exhaustion of $\mathscr{C}(S)$ by finite rigid sets. Here, $\operatorname{Mod}(S)$ denotes the index 2 subgroup of $\operatorname{Mod}^{ \pm}(S)$ consisting of those mapping classes that preserve orientation.

Proposition 3.13. Let $Y \subset \mathscr{C}(S)$ be a finite rigid set such that $\operatorname{Mod}(S) \cdot Y=\mathscr{C}(S)$. Suppose there exists $G \subset Y$ such that

(1) the $\operatorname{set}\left\{f_{\alpha} \mid \alpha \in G\right\}$ generates $\operatorname{Mod}(S)$, and

(2) $Y \cap f_{\alpha}(Y)$ is weakly rigid for all $\alpha \in G$.

Then there exists a sequence $Y=Y_{1} \subset Y_{2} \subset \cdots \subset Y_{n} \subset \cdots$ such that $Y_{i}$ is a finite rigid set, $Y_{i}$ has trivial pointwise stabilizer in $\operatorname{Mod}^{ \pm}(S)$ for all $i$, and

$$
\bigcup_{i \in \mathbb{N}} Y_{i}=\mathscr{b}(S) .
$$

Proof. First, the fact that $Y$ is rigid implies that $f_{\alpha}(Y)$ is rigid for all $\alpha \in Y$. Therefore, the set $Y_{2}:=Y \cup f_{G}(Y)$ is also rigid by assumption (2) and repeated application of Lemma 3.7. We now define, for all $n \geq 2$,

$$
Y_{n+1}:=Y_{n} \cup f_{G}\left(Y_{n}\right) .
$$

By induction, we see that $Y_{n}$ is rigid for all $n$ and so the first claim follows. Next, the pointwise stabilizer of $Y$ in $\operatorname{Mod}^{ \pm}(S)$ is trivial because $Y \cap f_{\alpha}(Y)$ is weakly rigid. Therefore, $Y_{n}$ has trivial pointwise stabilizer in $\operatorname{Mod}^{ \pm}(S)$, as $Y \subset Y_{n}$ for all $n$. Finally, since $\left\{f_{\alpha} \mid \alpha \in G\right\}$ generates $\operatorname{Mod}(S)$ and $\operatorname{Mod}(S) \cdot Y=\mathscr{C}(S)$, it follows that

$$
\bigcup_{i \in \mathbb{N}} Y_{i}=\mathscr{C}(S)
$$

which completes the proof.

We end this section by explaining how Theorem 1.1 implies that curve complexes are simplicially rigid

Proof of Corollary 1.2. Let $S \neq S_{1,2}$, and let $\phi: \mathscr{C}(S) \rightarrow \mathscr{C}(S)$ be a locally injective simplicial map. Let $\mathfrak{X}_{1} \subset \mathfrak{X}_{2} \subset \cdots$ be the exhaustion of $\mathscr{C}(S)$ provided by Theorem 1.1. Since $\mathfrak{X}_{i}$ is rigid and has trivial pointwise stabilizer in $\operatorname{Mod}^{ \pm}(S)$, there exists a unique mapping class $h_{i} \in \operatorname{Mod}^{ \pm}(S)$ such that $\left.h_{i}\right|_{\mathfrak{x}_{i}}=\phi \mid \mathfrak{x}_{i}$. Finally, Lemma 3.7 implies that $h_{i}=h_{j}$ for all $i, j$, and thus the result follows.

\section{Punctured spheres}

In this section we prove Theorem 1.1 for $S=S_{0, n}$. If $n \leq 3$ then $\mathscr{C}(S)$ is empty and thus the result is trivially true. The case $n=4$ is dealt with at the end of this section, as it needs special treatment. Thus, from now on we assume that $n \geq 5$. As in [AL] we represent $S$ as the double of an $n$-gon $\Delta$ with vertices removed, and define $\mathfrak{X}$ as 


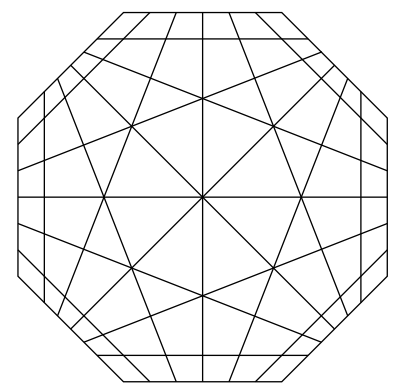

Figure 2. Octagon and arcs for $S_{0,8}$.

the set of curves on $S$ obtained by connecting every nonadjacent pair of sides of $\Delta$ by a straight line segment and then doubling; see Figure 2 for the case $n=8$.

Note that the pointwise stabilizer of $\mathfrak{X}$ in $\operatorname{Mod}^{ \pm}(S)$ has order two, and is generated by an orientation-reversing involution $i: S \rightarrow S$ that interchanges the two copies of $\Delta$. The rigidity of the set $\mathfrak{X}$, which was established in [AL], may be rephrased as follows:

Theorem 4.1 [AL]. For any locally injective simplicial map $\phi: \mathfrak{X} \rightarrow \mathscr{C}(S)$, there exists a unique $h \in \operatorname{Mod}(S)$ such that $\left.h\right|_{\mathfrak{X}}=\phi$, unique up to precomposing with $i$.

We are going to enlarge the set $\mathfrak{X}$ in the fashion described in Section 3 . We number the sides of $\Delta$ in a cyclic order, and denote by $\alpha_{j}$ the curve defined by the arc on $\Delta$ that connects the sides with labels $j$ and $j+2 \bmod n$. Let $A=\left\{\alpha_{1}, \ldots, \alpha_{n}\right\}$; in the terminology of [AL], $A$ is the set of chain curves of $\mathfrak{X}$. Observe that every element of $A$ bounds a disk containing exactly two punctures of $S$, and that if two elements of $A$ have nonzero intersection number then they are Farey neighbors in $\mathfrak{X}$. Thus we see that $A$ is a closed string of $n$ twistable Farey neighbors, and may consider the set $\mathfrak{X}_{A}$ from Definition 3.10. As a first step towards proving Theorem 1.1 for $S_{0, n}$, we show that $\mathfrak{X}_{A}$ is rigid. Since the pointwise stabilizer of $\mathfrak{X}_{A}$ is trivial, this amounts to the following statement:

Theorem 4.2. For any locally injective simplicial map $\phi: \mathfrak{X}_{A} \rightarrow \mathscr{C}(S)$, there exists a unique $g \in \operatorname{Mod}^{ \pm}(S)$ such that $\left.g\right|_{\mathfrak{X}_{A}}=\phi$.

Proof. Let $\phi: \mathfrak{X}_{A} \rightarrow \mathscr{C}(S)$ be a locally injective simplicial map. By Theorem 4.1, there exists $h \in \operatorname{Mod}^{ \pm}(S)$ such that $\left.h\right|_{\mathfrak{X}}=\left.\phi\right|_{\mathfrak{X}}$, unique up to precomposing with the involution $i$. Since $i$ fixes every element of $\mathfrak{X}$, after precomposing $\phi$ with $h^{-1}$ we may assume that $\left.\phi\right|_{\mathfrak{X}}$ is the identity map. We have $\phi\left(\mathfrak{X}_{A}\right)=\mathfrak{X}_{A}$ by Proposition 3.12; moreover, the automorphism group of $\mathfrak{X}_{A}$ fixing $\mathfrak{X}$ pointwise has order two, generated by the involution $\sigma: \mathfrak{X}_{A} \rightarrow \mathfrak{X}_{A}$ that interchanges $f_{\alpha_{i}}\left(\alpha_{i+1}\right)$ and $f_{\alpha_{i+1}}^{-1}\left(\alpha_{i}\right)$ for all $i$. Since $\left.i\right|_{\mathfrak{X}_{A}}=\sigma$, up to precomposing $\phi$ with $i$, we deduce that $\left.\phi\right|_{\mathfrak{X}_{A}}$ is the identity, as we wanted to prove. 
We now prove Theorem 1.1 for spheres with punctures:

Proof of Theorem 1.1 for $S=S_{0, n}, n \geq 5$. Let $\mathfrak{X}_{A}$ be the set constructed above, which is rigid and has trivial pointwise stabilizer in $\operatorname{Mod}^{ \pm}(S)$, by Theorem 4.2. The set $\left\{H_{\alpha} \mid \alpha \in A\right\}$ generates $\operatorname{Mod}(S)$; see, for instance, Corollary 4.15 of [Farb and Margalit 2012]. In addition, $\mathfrak{X}_{A} \cap H_{\alpha}\left(\mathfrak{X}_{A}\right)$ is weakly rigid for all $\alpha \in A$, as it contains $A$ and $H_{\alpha_{i}}\left(\alpha_{j}\right)$ for any $\alpha_{i}, \alpha_{j}$ disjoint from $\alpha$. Finally, by inspection we see $\operatorname{Mod}(S) \cdot \mathfrak{X}_{A}=\mathscr{C}(S)$. Therefore, we may apply Proposition 3.13 to the sets $Y=\mathfrak{X}_{A}$ and $G=A$ to obtain the desired sequence $\mathfrak{X}_{A}=Y_{1} \subset Y_{2} \subset \cdots \subset Y_{n} \subset \cdots$ of finite rigid sets.

Proof of Theorem 1.1 for $S=S_{0,4}$. As mentioned in the introduction, in this case $\mathscr{C}(S)$ is isomorphic to the Farey complex. It is easy to see, and is otherwise explicitly stated in [AL], that any triangle in $\mathscr{C}(S)$ is rigid. From this, plus the fact that any edge in $\mathscr{C}(S)$ is contained in exactly two triangles, it follows that any subcomplex of $\mathscr{C}(S)$ that is homeomorphic to a disk is also rigid. Consider the dual graph of $\mathscr{C}(S)$ (which is in fact a trivalent tree $T$ ), equipped with the natural path metric. Let $Y_{1}$ be a triangle in $\mathscr{C}(S)$, and define $Y_{n}$ to be the union of all triangles of $\mathscr{C}(S)$ whose corresponding vertices in $T$ are at distance at most $n$ from the vertex corresponding to $Y_{1}$. Then the sequence $\left(Y_{n}\right)_{n \in \mathbb{N}}$ gives the desired exhaustion of $\mathscr{C}(S)$.

\section{Closed and punctured surfaces of genus $g \geq 2$}

In this section we consider the case of a surface $S$ of genus $g \geq 2$ with $n \geq 0$ marked points. First observe that if $g=2$ and $n=0$, then since $\mathscr{C}\left(S_{2,0}\right) \cong \mathscr{C}\left(S_{0,6}\right)$ [Luo 2000], the main theorem for $S_{2,0}$ follows from the case $S_{0,6}$, already proved in Section 4 . We therefore assume that $n \geq 1$ if $g=2$. Once we have recalled some properties of $\mathfrak{X} \subset \mathscr{C}(S)$ from [AL], we sketch the proof of Theorem 1.1 for closed surfaces as it is simpler.

We let $\mathfrak{X} \subset \mathscr{C}(S)$ denote the finite rigid set constructed in [AL]. The definition of the set $\mathfrak{X}$ is somewhat involved and we will not recall it in full detail. Instead, we first note that $\mathfrak{X}$ contains the set of chain curves

$$
\mathfrak{C}=\left\{\alpha_{0}^{0}, \ldots, \alpha_{0}^{n}, \alpha_{1}, \ldots, \alpha_{2 g+1}\right\}
$$

depicted in Figure 3. For notational purposes we also write $\alpha_{0}=\alpha_{0}^{1}$ (or in case $n=0, \alpha_{0}=\alpha_{0}^{0}$ ). In addition to these curves, $\mathfrak{X}$ contains every curve which occurs as the boundary component of a subsurface of $S$ filled by a subset $A \subset \mathfrak{C}$, provided its union is connected in $S$ and has one of the following forms:

(1) $A=\left\{\alpha_{0}^{i}, \alpha_{0}^{j}, \alpha_{k}\right\}$ where $0 \leq i \leq j \leq n$ and $k=1$ or $2 g+1$.

(2) $A=\left\{\alpha_{0}^{i}, \alpha_{0}^{j}, \alpha_{k}, \alpha_{k+1}\right\}$ where $0 \leq i \leq j \leq n$ and $k=1$ or $2 g$. 
(3) $A=\left\{\alpha_{i} \mid i \in I\right\}$ where $I \subset\{0, \ldots, 2 g+1\}$ is an interval (modulo $2 g+2$ ). If $n>0$ and $A$ has an odd number of curves, then we additionally require that the first and last numbers in the interval $I$ be even.

See Figure 4 for some key examples.

The pointwise stabilizer of $\mathfrak{X}$ in $\operatorname{Mod}^{ \pm}(S)$ is trivial. Thus the rigidity of the set $\mathfrak{X}$, established in [AL], may be rephrased as follows.

Theorem 5.1 [AL]. Let $S=S_{g, n}$ with $g \geq 2$ and $n \geq 0$ (and $n \geq 1$ if $g=2$ ). For any locally injective simplicial map $\phi: \mathfrak{X} \rightarrow \mathscr{C}(S)$, there exists a unique $h \in \operatorname{Mod}^{ \pm}(S)$ such that $\left.h\right|_{\mathfrak{X}}=\phi$.

Sketch of Theorem 1.1 for closed surfaces. Since the closed case avoids some of the technicalities that arise in the general case, we sketch the proof here. We begin by noting that in $[\mathrm{AL}]$ it is shown that $\mathfrak{X}$ contains every curve which occurs as the boundary component of a subsurface of $S$ filled by a subset $A \subset \mathfrak{C}$, provided its union is connected in $S$, without any further qualifications on the set $A$.

We enlarge $\mathfrak{X}$ to $\mathfrak{X}^{2}=\left(\mathfrak{X}^{\prime}\right)^{\prime}$ and consider the set $\mathfrak{X}^{2} \cup T_{\mathfrak{C}}(\mathfrak{C})$. The set $\mathfrak{C}$ is a closed string of twistable Farey neighbors (that the nonzero intersections are $\mathfrak{X}^{2}$-detectable follows from their $\mathfrak{X}$-detectability proved in $[\mathrm{AL}]$ ). By Proposition 3.12, this set will be rigid if we can rule out the potential order two symmetry. It thus suffices to show that one of the curves in $T_{\mathfrak{C}}(\mathfrak{C})$ is already in $\mathfrak{X}^{2}$. This is illustrated for $T_{\alpha_{2 g}}\left(\alpha_{2 g-1}\right)$ in Figure 8 (the pictures for a closed surface are obtained by ignoring punctures and any curves which subsequently become trivial, and identifying pairs

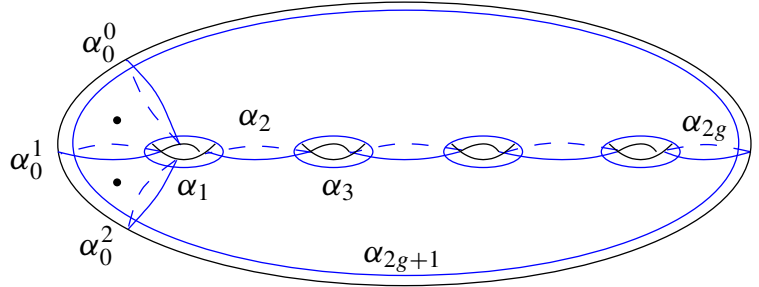

Figure 3. Chain curves $\mathfrak{C}$ on a genus 4 surface with 2 marked points.

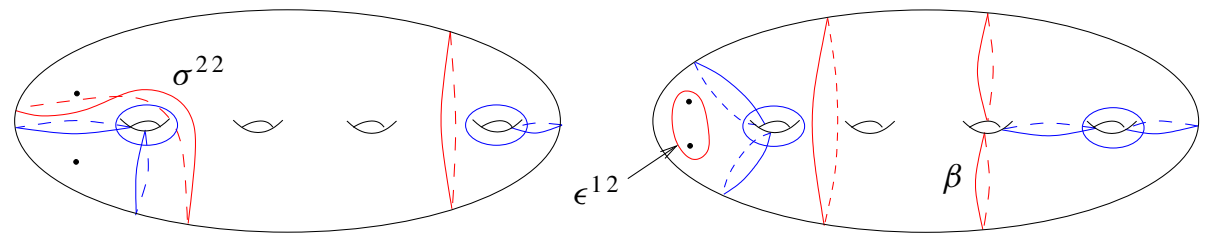

Figure 4. Examples of subsets of $\mathfrak{C}$ (in blue), together with the boundary components (in red) of the subsurface filled by them. The red curves are in $\mathfrak{X}$. 
that become isotopic). Therefore, $\mathfrak{X}^{2} \cup \mathcal{T}_{\mathfrak{C}}(\mathfrak{C})$ is indeed rigid. One can also find an appropriate closed string of twistable Farey neighbors containing the curve $\beta$ shown in Figure 4 (see Lemma 5.5 below), and so it follows that $\mathfrak{X}^{2} \cup T_{\mathfrak{C}}(\mathfrak{C}) \cup T_{\beta}(\mathfrak{C})$ is also rigid. Since $T_{\alpha_{0}}, \ldots, T_{\alpha_{2 g+1}}, T_{\beta}$ generate $\operatorname{Mod}(S)$ (see, e.g., [Farb and Margalit 2012]), Theorem 1.1 follows from Proposition 3.13.

The general case. It will be necessary to refer to some of the curves in $\mathfrak{X}$ by name, so we describe the naming convention briefly in those cases, along the lines of [AL]. We have already described the names of the elements of $\mathfrak{C}$. For $0<i<j \leq n$ we let $\epsilon^{i j}$ be the boundary component of the subsurface $N\left(\alpha_{1} \cup \alpha_{0}^{i-1} \cup \alpha_{0}^{j}\right)$ that also bounds a $(j-i+1)$-punctured disk in $S$ (containing the $i$-th through $j$-th punctures). We call the curves $\epsilon^{i j}$ outer curves; see Figure 4. For $0<i \leq j \leq n$, we also consider the other boundary component of $N\left(\alpha_{1} \cup \alpha_{0}^{i-1} \cup \alpha_{0}^{j}\right)$; this is a separating curve dividing the surface into two (punctured) subsurfaces of genus 1 and $g-1$ respectively. We denote this curve $\sigma^{i j}$. One more curve in $\mathfrak{X}$ that we refer to as $\beta$ is shown in Figure 4, and is a component of the boundary of the subsurface $N\left(\alpha_{2 g-2} \cup \alpha_{2 g-1} \cup \alpha_{2 g}\right)$.

The strategy for proving Theorem 1.1 for surfaces of genus $g \geq 2$ is similar in spirit to the one for punctured spheres, although considerably more involved. The main idea is to produce successive rigid enlargements of the rigid set $\mathfrak{X}$ identified in [AL], until we are in a position to apply Proposition 3.13. We begin by replacing $\mathfrak{X}$ with $\mathfrak{X}^{\prime}$, which is rigid by Proposition 3.5. For every $0<j \leq n$, let

$$
A_{j}=\left\{\sigma^{i j} \mid 0<i \leq j\right\} \cup\left\{\sigma^{j i} \mid j \leq i \leq n\right\} \cup\left\{\alpha_{1}, \alpha_{3}, \alpha_{4}, \alpha_{5}, \ldots, \alpha_{2 g+1}\right\} .
$$

The set $A_{j}$ is almost filling and uniquely determines a curve denoted $\alpha_{1}^{j}$; see Figure 5. The naming is suggestive, as all $\alpha_{1}^{j}$ are homotopic to $\alpha_{1}$ upon filling in the punctures.

We can similarly find a subset $A_{0}$ (shown in the left of Figure 6) which is almost filling and uniquely determines a curve denoted $\alpha_{1}^{0}$ (shown on the right of Figure 6), which bounds a disk enclosing every puncture of $S$. Consequently, $\alpha_{1}^{j} \in \mathfrak{X}^{\prime}$ for all $j=0, \ldots, n$.
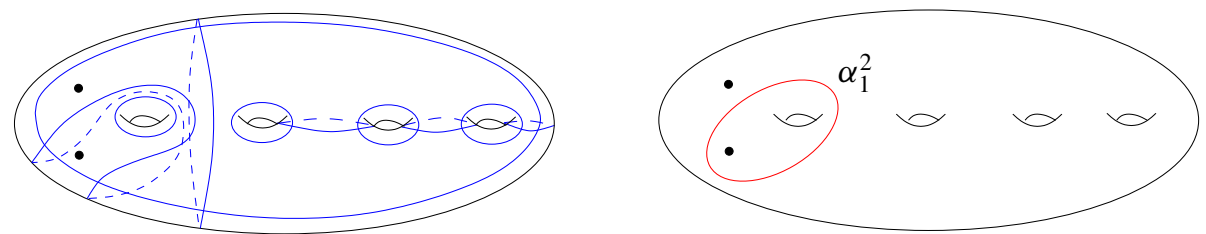

Figure 5. The surface on the left contains the set $A_{2}$ which uniquely determines $\alpha_{1}^{2}$. 

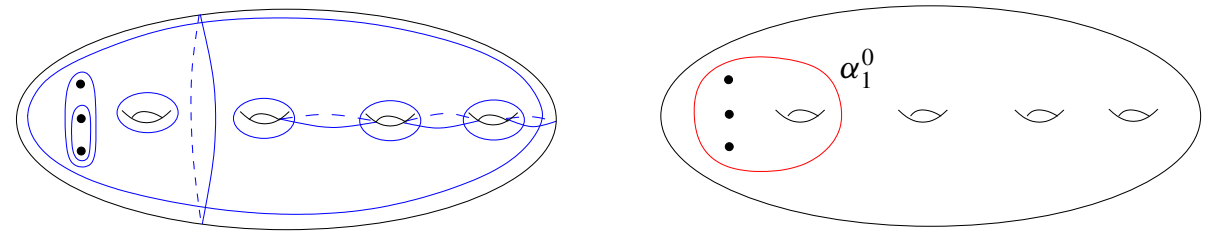

Figure 6. The curves $A_{0} \subset \mathfrak{X}$ (left) and the curve $\alpha_{1}^{0} \in \mathfrak{X}^{\prime}$ (right).
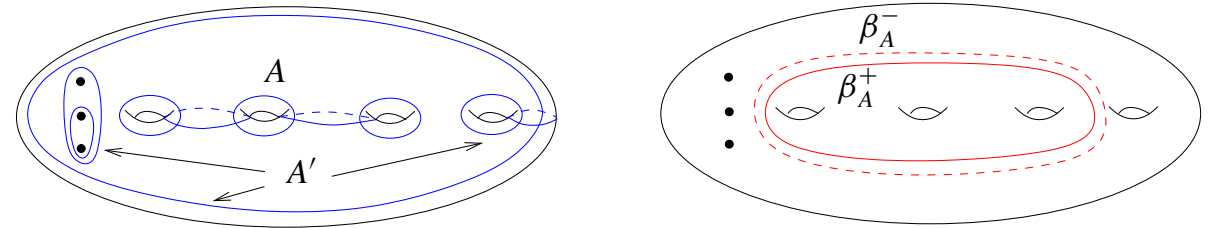

Figure 7. The sets $A=\left\{\alpha_{1}, \ldots, \alpha_{5}\right\} \subset \mathfrak{C}$ and $A^{\prime} \subset \mathfrak{X}$ (left) and the curves $\beta_{A}^{ \pm} \in \mathfrak{X}^{\prime}$ determined by $A \cup A^{\prime}$ (right).

Punctured surface promotion. One issue that arises only in the case $n>0$ is that for intervals $I \subset\{0, \ldots, 2 g+1\}$ (modulo $2 g+2$ ) of odd length, the boundary curves of the neighborhood of the subsurface filled by $A=\left\{\alpha_{i} \mid i \in I\right\}$ are only contained in $\mathfrak{X}$ when $I$ starts and ends with even indexed curves. Passing to the set $\mathfrak{X}^{\prime}$ allows us to easily enlarge further to a set which rectifies this problem.

Specifically, we define $\mathfrak{X}_{1}$ to be the union of $\mathfrak{X}^{\prime}$ together with boundary components of subsurfaces filled by sets $A=\left\{\alpha_{i}, \alpha_{i+1}, \ldots, \alpha_{j}\right\}$ where $0<i \leq j \leq 2 g-1$ and $i, j$ are both odd. See Figure 7 for examples. Let $\mathfrak{B}_{o}$ be the set of all curves defined by such sets $A$.

Before we proceed, we describe this set in more detail. Cutting $S$ open along $\alpha_{1} \cup \alpha_{3} \cup \cdots \cup \alpha_{2 g-1} \cup \alpha_{2 g+1}$ we obtain two components $\Theta_{o}^{+}$and $\Theta_{o}^{-}$. These are each spheres with holes: $\Theta_{o}^{+}$is the sphere in "front" in Figure 3, which is a $(g+n+1)$ holed sphere containing the $n$ punctures of $S$, while $\Theta_{o}^{-}$is the $(g+1)$-holed sphere in the "back" in Figure 3. For every $A=\left\{\alpha_{i}, \alpha_{i+1}, \ldots, \alpha_{j}\right\}$ where $0<i<j \leq 2 g-1$ and $i, j$ are both odd, the boundary of the subsurface filled by $A$ has exactly two components $\beta_{A}^{ \pm}$with $\beta_{A}^{+} \subset \Theta_{o}^{+}$and $\beta_{A}^{-} \subset \Theta_{o}^{-}$(possibly peripheral in $\Theta_{o}^{ \pm}$depending on $A$ ). Furthermore, for every such set $A$, there is a "complementary" set $A^{\prime} \subset \mathfrak{X}$ such that $A \cup A^{\prime}$ is almost filling, and such that $\left\{\beta_{A}^{ \pm}\right\}$is the set determined by $A \cup A^{\prime}$. See Figure 7.

Lemma 5.2. For all $g \geq 2$ and $n \geq 1$, the set $\mathfrak{X}_{1}$ is rigid and has trivial pointwise stabilizer in $\operatorname{Mod}^{ \pm}\left(S_{g, n}\right)$.

Proof. First, $\mathfrak{X}_{1}$ has trivial pointwise stabilizer since $\mathfrak{X}$ does. Given any locally injective simplicial map $\phi: \mathfrak{X}_{1} \rightarrow \mathscr{C}(S)$, there exists a unique $h \in \operatorname{Mod}^{ \pm}(S)$ such 
that $\phi=\left.h\right|_{\mathfrak{X}^{\prime}}$, by Theorem 5.1 and Proposition 3.5. Composing with the inverse of $h$ if necessary, we can assume $\phi$ is the identity on $\mathfrak{X}^{\prime}$. So we need only show that $\phi(\gamma)=\gamma$ for all $\gamma \in \mathfrak{X}_{1}-\mathfrak{X}^{\prime}$. With respect to the notation above, any such curve is $\beta_{A}^{ \pm}$for $A=\left\{\alpha_{i}, \alpha_{i+1}, \ldots, \alpha_{j}\right\}$, where $0<i \leq j \leq 2 g-1$ and $i, j$ are both odd. Since $A \cup A^{\prime}$ is almost filling, $\phi\left(\left\{\beta_{A}^{ \pm}\right\}\right)=\left\{\beta_{A}^{ \pm}\right\}$. Now, for $A=\left\{\alpha_{1}, \alpha_{2}, \alpha_{3}\right\}$, we have $i\left(\beta_{A}^{+}, \alpha_{0}^{1}\right) \neq 0$ and $i\left(\beta_{A}^{-}, \alpha_{0}^{1}\right)=0$; here, $\alpha_{0}^{1}$ is the curve depicted in Figure 6 . Therefore $\phi\left(\beta_{A}^{+}\right)=\beta_{A}^{+}$, as $\phi$ is locally injective and simplicial. Finally, an easy connectivity argument involving the set of curves $\left\{\beta_{A}^{ \pm}\right\}_{A}$ yields the desired result.

Half the proof and the case of one or fewer punctures. We now enlarge the set $\mathfrak{X}_{1} \subset \mathscr{C}(S)$ from Lemma 5.2 to $\mathfrak{X}_{1}^{2}=\left(\mathfrak{X}_{1}^{\prime}\right)^{\prime} \subset \mathscr{C}(S)$. According to Proposition 3.5, $\mathfrak{X}_{1}^{2}$ is rigid, and since the pointwise stabilizer of $\mathfrak{X}$ is trivial, so is the pointwise stabilizer of $\mathfrak{X}_{1}^{2}$. We will need the following lemma; see Figure 3 for the labeling of the curves.

Lemma 5.3. For any $g \geq 2$ and $n \geq 0$ (with $n \geq 1$ if $g=2$ ), we have $T_{\alpha_{2 g}}\left(\alpha_{2 g-1}\right) \in \mathfrak{X}_{1}^{2}$. Proof. This requires a series of pictures, slightly different for the case $g \geq 3$ and for $g=2$.

Case 1: $g \geq 3$. We refer the reader to Figure 8 ; although we have only drawn the figures for $g=3$ and $n=2$, it is straightforward to extend them to all $g \geq 3$ and $n \geq 0$. The upper left figure shows an almost filling set of curves contained in $\mathfrak{X}_{1}$, determining uniquely the curve on the upper right figure, which is thus in $\mathfrak{X}_{1}^{\prime}$. This curve is then used to produce an almost filling set, depicted on the lower left hand figure, that uniquely determines $T_{\alpha_{2 g}}\left(\alpha_{2 g-1}\right)$, shown on the right. Thus we see that $T_{\alpha_{2 g}}\left(\alpha_{2 g-1}\right) \in \mathfrak{X}_{1}^{2}$, as claimed.
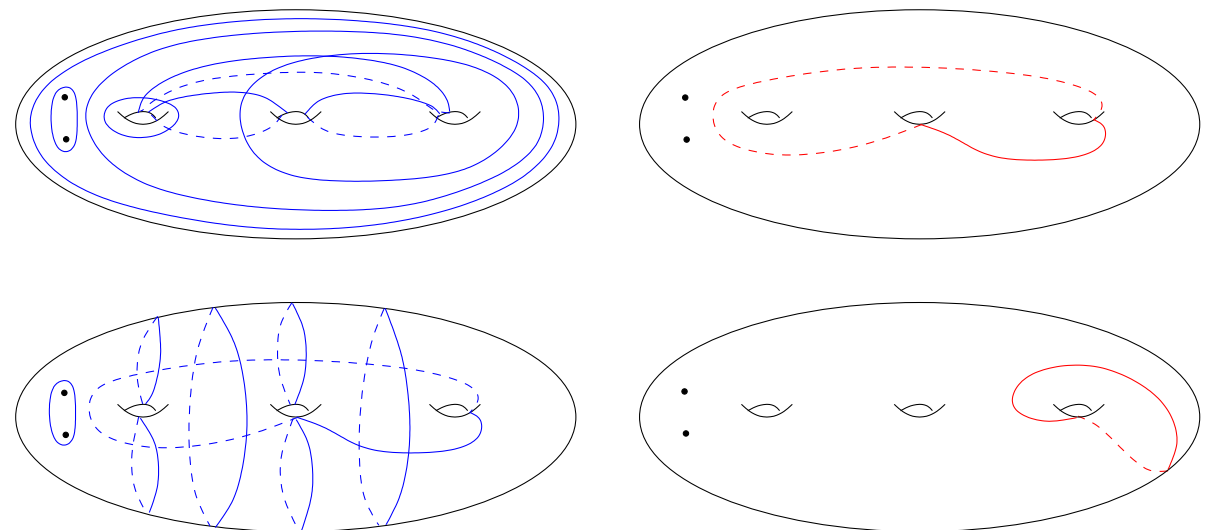

Figure 8. Illustrating $T_{\alpha_{2 g}}\left(\alpha_{2 g-1}\right)$ in $\mathfrak{X}_{1}^{2}$, when $g=3$. The almost filling set on the left (blue) uniquely determines the curve in the right figure (red). 

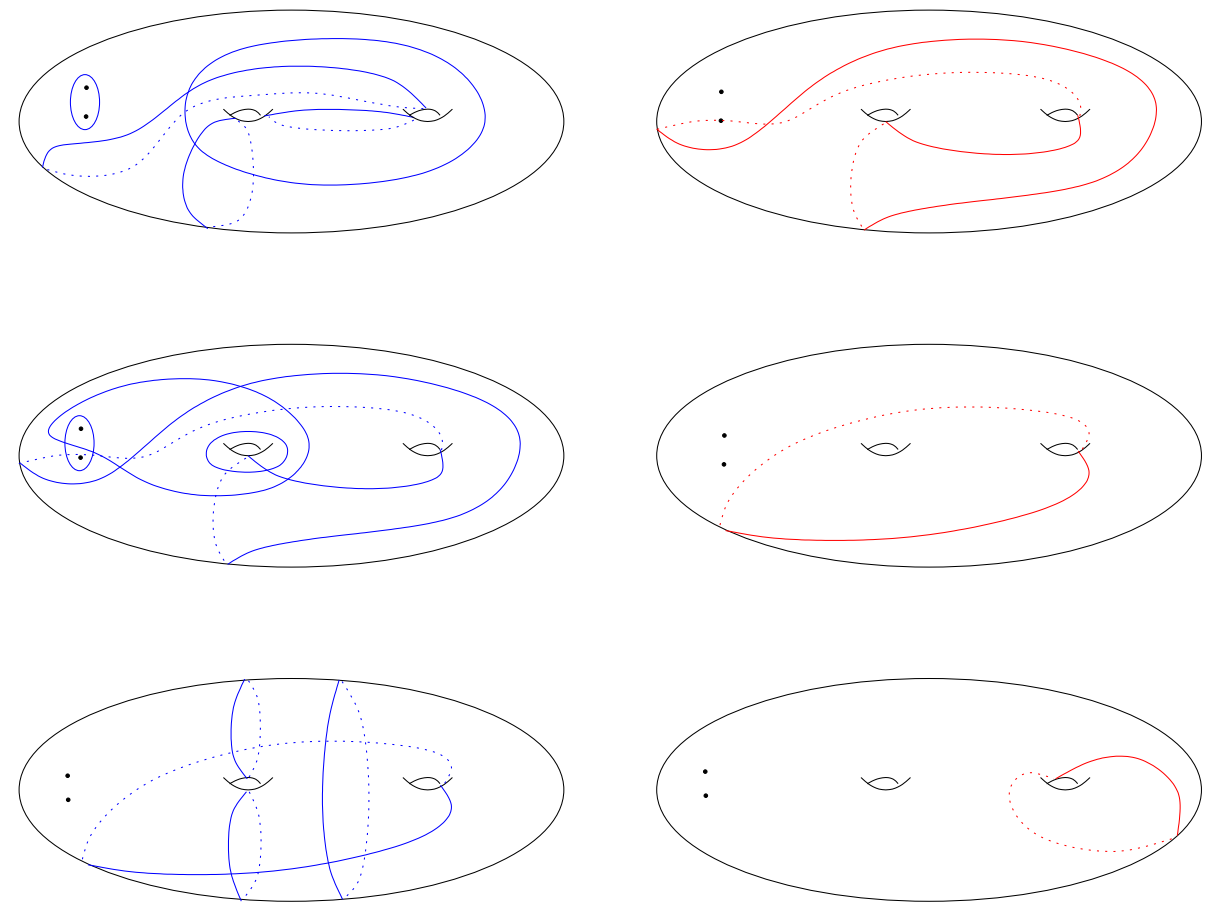

Figure 9. Illustrating $T_{\alpha_{2 g}}\left(\alpha_{2 g-1}\right)$ in $\mathfrak{X}_{1}^{2}$, when $g=2$ and $n \geq 1$. The almost filling set on the left (blue) uniquely determines the curve in the right figure (red).

Case 2: $g=2$ and $n \geq 1$. In this case a different set of pictures is required; see Figure 9. The upper left hand figure shows an almost filling set of curves that is contained in $\mathfrak{X}_{1}$ and uniquely determines the curve shown on the upper right. This curve is then used to produce an almost filling set, depicted in the middle left picture, which is contained in $\mathfrak{X}_{1}^{\prime}$ and uniquely determines the curve in the middle right figure. We now make use of this new curve to produce an almost filling set (lower left) that is contained in $\mathfrak{X}_{1}^{2}$ and uniquely determines $T_{\alpha_{2 g}}\left(\alpha_{2 g-1}\right)$ (lower right).

We claim that the set $\mathfrak{X}_{1}^{2} \cup T_{\mathfrak{C}}(\mathfrak{C})$ is rigid. More concretely:

Lemma 5.4. Let $\phi: \mathfrak{X}_{1}^{2} \cup T_{\mathfrak{C}}(\mathfrak{C}) \rightarrow \mathscr{C}(S)$ be a locally injective simplicial map. Then there exists a unique $h \in \operatorname{Mod}^{ \pm}(S)$ such that $\left.h\right|_{\mathfrak{X}_{1}^{2} \cup T_{\mathfrak{C}}(\mathfrak{C})}=\phi$.

Proof. Let $\phi: \mathfrak{X}_{1}^{2} \cup T_{\mathfrak{C}}(\mathfrak{C})$ be a locally injective simplicial map. Since $\mathfrak{X}_{1}^{2}$ is rigid and its pointwise stabilizer in $\operatorname{Mod}^{ \pm}(S)$ is trivial, there exists a unique $h \in \operatorname{Mod}^{ \pm}(S)$ such that $\left.h\right|_{\mathfrak{X}_{1}^{2}}=\left.\phi\right|_{\mathfrak{X}_{1}^{2}}$. Precomposing $\phi$ with $h^{-1}$, we may assume that in fact $\left.\phi\right|_{\mathfrak{X}_{1}^{2}}$ is the identity map.

For $i=0, \ldots, n, \mathfrak{C}_{i}=\left\{\alpha_{0}^{i}, \alpha_{1}, \ldots, \alpha_{2 g+1}\right\}$ is a closed string of twistable Farey neighbors in $\mathfrak{X}_{1}^{2}$ (the fact that the nonzero intersection numbers between these curves 
is $\mathfrak{X}$-detectable, hence $\mathfrak{X}_{1}^{2}$-detectable, is shown in the proofs of Theorem 5.1 and 6.1 in $[\mathrm{AL}])$. Consider the set $\mathfrak{X}_{1}^{2} \cup T_{\mathfrak{C}_{i}}\left(\mathfrak{C}_{i}\right)$, and observe that, in the terminology of Definition 3.10, it equals $Y_{A}$ for $Y=\mathfrak{X}_{1}^{2}$ and $A=\mathfrak{C}_{i}$. By Proposition 3.12,

$$
\phi\left(\mathfrak{X}_{1}^{2} \cup T_{\mathfrak{C}_{i}}\left(\mathfrak{C}_{i}\right)\right)=\mathfrak{X}_{1}^{2} \cup T_{\mathfrak{C}_{i}}\left(\mathfrak{C}_{i}\right) ;
$$

moreover, the automorphism group of $\mathfrak{X}_{1}^{2} \cup T_{\mathfrak{C}_{i}}\left(\mathfrak{C}_{i}\right)$ fixing $\mathfrak{X}_{1}^{2}$ pointwise has order at most two. But, by Lemma 5.3, $T_{\alpha_{2 g}}\left(\alpha_{2 g-1}\right) \in \mathfrak{X}_{1}^{2}$ and thus this group is trivial. In other words, we have shown that the set $\mathfrak{X}_{1}^{2} \cup T_{\mathfrak{C}_{i}}\left(\mathfrak{C}_{i}\right)$ is rigid.

Now, $\mathfrak{X}_{1}^{2} \cup T_{\mathfrak{C}_{0}}\left(\mathfrak{C}_{0}\right) \cup T_{\mathfrak{C}_{1}}\left(\mathfrak{C}_{1}\right)$ is also rigid by Lemma 3.7, since

$$
\left(\mathfrak{X}_{1}^{2} \cup T_{\mathfrak{C}_{0}}\left(\mathfrak{C}_{0}\right)\right) \cap\left(\mathfrak{X}_{1}^{2} \cup T_{\mathfrak{C}_{1}}\left(\mathfrak{C}_{1}\right)\right)
$$

is weakly rigid as it contains $\mathfrak{X}_{1}^{2}$. Since $T_{\mathfrak{C}}(\mathfrak{C})=\bigcup_{i=0}^{n} T_{\mathfrak{C}_{i}}\left(\mathfrak{C}_{i}\right)$, we may repeat essentially this same argument $n-1$ more times to conclude $\mathfrak{X}_{1}^{2} \cup T_{\mathfrak{C}}(\mathfrak{C})$ is rigid, as required.

Next, we provide a further enlargement of our rigid set. Let $\beta$ be the curve depicted in Figure 4, which is one of the boundary components of the surface $N\left(\alpha_{2 g-2} \cup \alpha_{2 g-1} \cup \alpha_{2 g}\right)$. We claim:

Lemma 5.5. The set $\mathfrak{X}_{1}^{2} \cup T_{\mathfrak{C}}(\mathfrak{C}) \cup T_{\beta}(\mathfrak{C})$ is rigid.

Proof. Let $\phi: \mathfrak{X}_{1}^{2} \cup T_{\mathfrak{C}}(\mathfrak{C}) \cup T_{\beta}(\mathfrak{C}) \rightarrow \mathscr{C}(S)$ be a locally injective simplicial map. By Lemma 5.4, $\mathfrak{X}_{1}^{2} \cup T_{\mathfrak{C}}(\mathfrak{C})$ is rigid and thus, up to precomposing $\phi$ with an element of $\operatorname{Mod}^{ \pm}(S)$, we may assume that $\left.\phi\right|_{\mathfrak{X}_{1}^{2} \cup T_{\mathfrak{C}}(\mathfrak{C})}$ is the identity. The set

$$
A=\left\{\alpha_{2 g}, \alpha_{2 g-1}, \alpha_{2 g-2}, \beta, \alpha_{2 g+1}\right\} \subset \mathfrak{X}_{1}^{2}
$$

is a closed string of twistable Farey neighbors in $\mathfrak{X}_{1}^{2}$ (again, detectability of the nonzero intersection numbers is shown in [AL]). Therefore, we may apply Proposition 3.12 to $\mathfrak{X}=\mathfrak{X}_{1}^{2} \cup T_{\mathfrak{C}}(\mathfrak{C})$ and $A$ to deduce that $\phi\left(\mathfrak{X}_{A}\right)=\mathfrak{X}_{A}$; observe that $\mathfrak{X}_{A}=\mathfrak{X}_{1}^{2} \cup T_{\mathfrak{C}}(\mathfrak{C}) \cup T_{\beta}(\mathfrak{C})$. Moreover, the automorphism group of $\mathfrak{X}_{A}$ fixing $\mathfrak{X}$ pointwise is trivial, by Lemma 5.4, and thus the result follows.

Proof of Theorem 1.1 for $g \geq 2$ and $n \leq 1$. Let $Y=\mathfrak{X}_{1}^{2} \cup T_{\mathfrak{C}}(\mathfrak{C}) \cup T_{\beta}(\mathfrak{C})$. When $S$ is closed or has one puncture, the Dehn twists about chain curves and the Dehn twist about the curve $\beta$ generate $\operatorname{Mod}(S)$; see, for example, Corollary 4.15 of [Farb and Margalit 2012]. For $\gamma \in \mathfrak{C} \cup\{\beta\}$, the set

$$
T_{\gamma}(Y) \cap(Y)
$$

contains $\mathfrak{C}$, together with $T_{\alpha}\left(\alpha^{\prime}\right)$ for any $\alpha, \alpha^{\prime} \in \mathfrak{C}$ which are disjoint from $\gamma$. In particular, this set is weakly rigid. By inspection, the $\operatorname{Mod}(S)$-orbit of $Y$ is all of $\mathscr{C}(S)$, and so by Proposition 3.13, this set suffices to prove the theorem. 
Multiple punctures. When $S_{g, n}$ has $n \geq 2$ (and $g \geq 2$ ), the twists in the curves $\mathfrak{C}$ and $\{\beta\}$ do not generate the entire mapping class group. In this case, one needs to add the set of half-twists about the outer curves $\epsilon^{i(i+1)}$ bounding twice-punctured disks; see again Corollary 4.15 of [Farb and Margalit 2012]. Because of this, and in light of Proposition 3.13, when $n \geq 2$ we would like to enlarge our rigid set from the previous subsection by adding half-twists of chain curves about outer curves $\epsilon^{i(i+1)}$. In fact, denoting this set of outer curves by $\mathfrak{O}_{P}=\left\{\epsilon^{i(i+1)}\right\}_{i=1}^{n-1}$ we shall show that these curves are already in $\mathfrak{X}_{1}^{2}$. Specifically, we prove:

Lemma 5.6. We have $H_{\mathfrak{O}_{P}}(\mathfrak{C}) \subset \mathfrak{X}_{1}^{2}$.

Proof. If $\alpha \in \mathfrak{C}$ and $\epsilon^{j(j+1)} \in \mathfrak{O}_{P}$, then we must show that $H_{\epsilon^{j(j+1)}}(\alpha) \in \mathfrak{X}_{1}^{2}$ for each $j=1, \ldots, n-1$. This is clear if $i\left(\alpha, \epsilon^{j(j+1)}\right)=0$, since then $H_{\epsilon^{j(j+1)}}(\alpha)=\alpha$. The intersection number is nonzero only when $\alpha=\alpha_{0}^{j}$, so it suffices to consider only this case.

To prove $H_{\epsilon^{j(j+1)}}\left(\alpha_{0}^{j}\right) \in \mathfrak{X}_{1}^{2}$, we need only exhibit the almost filling sets from $\mathfrak{X}_{1}^{\prime}$ uniquely determining this curve. This in turn requires an almost filling set from $\mathfrak{X}_{1}$. As before, we provide the necessary curves in a sequence of two figures. First, the almost filling set on the left of Figure 10 is contained in $\mathfrak{X}^{\prime}$, and hence in $\mathfrak{X}_{1}$ (compare with Figure 5), and uniquely determines the curve $\gamma_{1}$ depicted on the right of the same figure. Therefore, $\gamma_{1} \in \mathfrak{X}_{1}^{\prime}$. Figure 11 is then an almost filling set in $\mathfrak{X}_{1}^{\prime}$, and uniquely determines the curve on the right of the same figure. This curve is $H_{\epsilon^{j(j+1)}}\left(\alpha_{0}^{j}\right)$, and so completes the proof.

We are finally in a position to prove Theorem 1.1 for surfaces of genus $g \geq 2$ and $n \geq 2$.
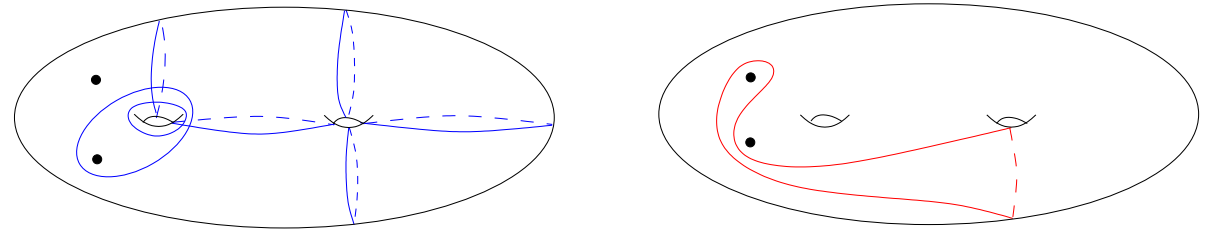

Figure 10. Determining the curve $\gamma_{1}$.
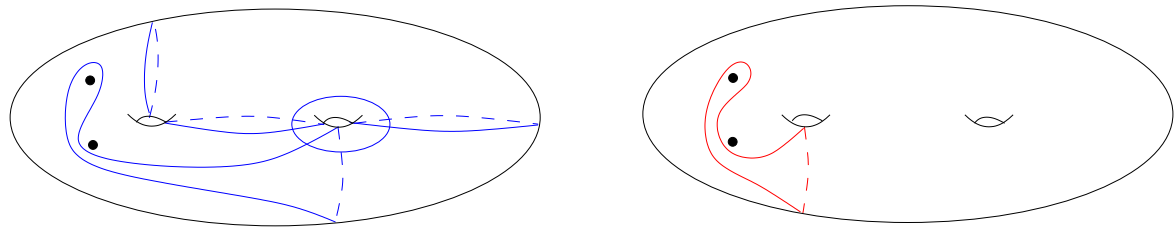

Figure 11. Determining the curve $H_{\epsilon^{i(i+1)}}\left(\alpha_{0}^{i}\right)$. 
Proof of Theorem 1.1 for $S=S_{g, n}, g \geq 2, n \geq 2$.. The set $Y=\mathfrak{X}_{1}^{2} \cup T_{\mathfrak{C}}(\mathfrak{C}) \cup T_{\beta}(\mathfrak{C})$ is rigid by Lemma 5.6, and has trivial pointwise stabilizer in $\operatorname{Mod}^{ \pm}(S)$ since $\mathfrak{X}$ does. Moreover, $\operatorname{Mod}(S) \cdot Y=\mathscr{C}(S)$ by inspection. Consider the subset $G=\mathfrak{C} \cup\{\beta\} \cup \mathfrak{O}_{P}$; as mentioned before, the (half-)twists about elements of $G$ generate $\operatorname{Mod}(S)$. In addition, for every $\alpha \in G, Y \cap f_{\alpha}(Y)$ is weakly rigid. Thus we can apply Proposition 3.13 to $Y$ and $G$, hence obtaining the desired exhaustion of $\mathscr{C}(S)$.

\section{Tori}

In this section we will prove Theorem 1.1 for $S=S_{1, n}$, for $n \geq 0$. First, if $n \leq 1$ then $\mathscr{C}(S)$ is isomorphic to the Farey complex, and thus the result follows as in the case of $S_{0,4}$; see Section 4. For $n=2$, Theorem 1.1 is not true as stated due to the existence of nongeometric automorphisms of $\mathscr{C}(S)$, as mentioned in the introduction. However, in light of the isomorphism $\mathscr{C}\left(S_{0,5}\right) \cong \mathscr{C}\left(S_{1,2}\right)$ [Luo 2000], the same statement holds after replacing the group $\operatorname{Mod}^{ \pm}(S)$ by $\operatorname{Aut}(\mathscr{C}(S))$ in the definition of rigid set, by the results of Section 4.

Therefore, from now on we assume $n \geq 3$. In [AL], we constructed a finite rigid set $\mathfrak{X}$ described as follows. View $S_{1, n}$ as a unit square with $n$ punctures along the horizontal midline and the sides identified. The set $\mathfrak{X}$ contains a subset $\mathfrak{C} \subset \mathfrak{X}$ of $n+1$ chain curves

$$
\mathfrak{C}=\left\{\alpha_{1}, \ldots, \alpha_{n}\right\} \cup\{\beta\}
$$

where $\alpha_{1}, \ldots, \alpha_{n}$ are distinct curves which appear as vertical lines in the square and $\beta$ is the curve which appears as a horizontal line; see Figure 12. We assume that the indices on the $\alpha_{i}$ are ordered cyclically around the torus, and that the punctures are labeled so that the $i$-th puncture lies between $\alpha_{i}$ and $\alpha_{i+1}$. The boundaries of the subsurfaces filled by connected unions of these chain curves form a collection
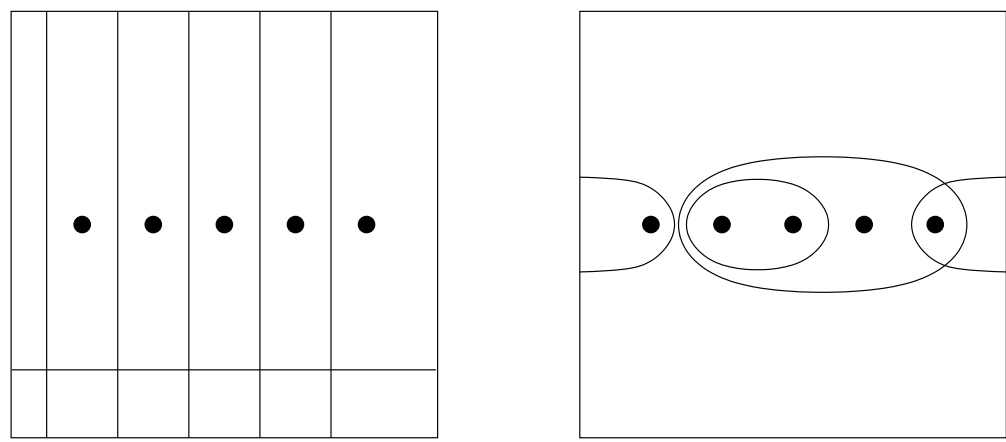

Figure 12. Chain curves on the left, and some examples of outer curves on the right, in $S_{1,5}$. 
of curves, denoted $\mathfrak{O}$ which we refer to as outer curves. Then

$$
\mathfrak{X}=\mathfrak{C} \cup \mathfrak{O} .
$$

This set has a nontrivial pointwise stabilizer in $\operatorname{Mod}^{ \pm}\left(S_{1, n}\right)$, which can be realized as the (descent to $S_{1, n}$ of the) horizontal reflection of the square through the midline containing the punctures. Denoting this involution $r: S_{1, n} \rightarrow S_{1, n}$, we summarize the result of $[\mathrm{AL}]$ in the following theorem.

Theorem 6.1 [AL]. For any locally injective simplicial map $\phi: \mathfrak{X} \rightarrow \mathscr{C}\left(S_{1, n}\right)$ there exists $h \in \operatorname{Mod}^{ \pm}\left(S_{1, n}\right)$ such that $\left.h\right|_{\mathfrak{X}}=\phi$. Moreover, $h$ is unique up to precomposing with $r$.

The strategy of proof is again similar to that of previous sections, although the technicalities are different, and boils down to producing an enlargement of the set $\mathfrak{X}$ so that Proposition 3.13 can be applied.

We begin by enlarging the set $\mathfrak{X}$ as follows. We let $\delta_{i}$ be the curve coming from the vertical line through the $i$-th puncture in the square. For every $1 \leq i \leq n$, let $\beta_{i}^{+}$be the curve obtained from $\beta$ by pushing it up over the $i$-th puncture. More precisely, we consider the point-pushing homeomorphism $f_{i}: S_{1, n} \rightarrow S_{1, n}$ that pushes the $i$-th puncture up and around $\delta_{i}$, and then let $\beta_{i}^{+}=f_{i}(\beta)$. We similarly define $\beta_{i}^{-}=f_{i}^{-1}(\beta)$, and set $\beta_{i(i+1)}^{ \pm}=f_{i+1}^{ \pm 1} f_{i}^{ \pm 1}(\beta)$, where the subscripts are taken modulo $n$. See Figure 13.

Let

$$
\mathfrak{X}_{1}=\mathfrak{X} \cup\left\{\beta_{i}^{ \pm} \mid 1 \leq i \leq n\right\} \cup\left\{\beta_{i(i+1)}^{ \pm} \mid 1 \leq i \leq n\right\}
$$

with indices in the last set taken modulo $n$. We first prove that this set is rigid; since the pointwise stabilizer of $\mathfrak{X}_{1}$ in $\operatorname{Mod}^{ \pm}\left(S_{1, n}\right)$ is trivial, this amounts to the following proposition.

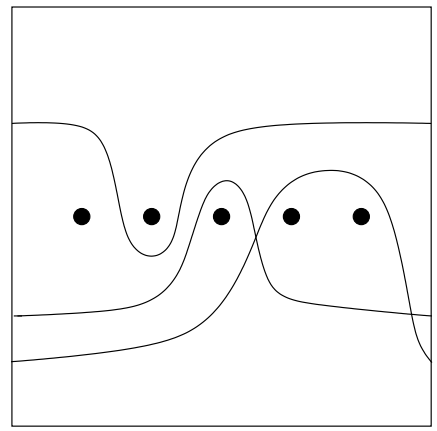

Figure 13. Curves $\beta_{2}^{-}, \beta_{3}^{+}$, and $\beta_{45}^{+}$on $S_{1,5}$. 
Proposition 6.2. For any locally injective simplicial map $\phi: \mathfrak{X}_{1} \rightarrow \mathscr{C}\left(S_{1, n}\right)$, there exists a unique $h \in \operatorname{Mod}^{ \pm}\left(S_{1, n}\right)$ so that $\left.h\right|_{\mathfrak{X}_{1}}=\phi$.

The proof of this proposition will require a repeated application of Lemma 2.4, and as such, we must verify that certain quadruples of curves satisfy the hypotheses of that lemma. We will need to refer to the outer curves by name. To this end, note that since any outer curve surrounds a set of (cyclically) consecutive punctures, we can determine an outer curve by specifying the first and last puncture surrounded. Consequently, we let $\epsilon^{i j}$ denote the outer curve surrounding all punctures from the $i$-th to the $j$-th, with all indices taken modulo $n$. Observe that since the set of punctures is cyclically ordered, we do not need to assume that $i<j$ in the definition of $\epsilon^{i j}$. We will need the following lemma.

Lemma 6.3. For each $1 \leq i \leq n$, consider the following four quadruples of curves in $\mathfrak{X}_{1}$, with indices taken modulo $n$ :

- $\beta_{(i-1) i}^{ \pm}, \epsilon^{(i+1) i}, \beta_{i}^{ \pm}, \epsilon^{(i-1) i}$,

- $\beta_{i(i+1)}^{ \pm}, \epsilon^{i(i-1)}, \beta_{i}^{ \pm}, \epsilon^{i(i+1)}$.

Each of these satisfies the hypothesis of Lemma 2.4. Furthermore, the nonzero intersections are all $\mathfrak{X}_{1}$-detectable. Consequently, $\epsilon^{(i+1) i}$ and $\epsilon^{i(i-1)}$ are the unique Farey neighbors of $\beta_{i}^{-}$and $\beta_{i}^{+}$.

Proof. The fact that the four quadruples of curves each satisfy the hypothesis of Lemma 2.4 is clear by inspection. See the left side of Figure 14 for the case

$$
\beta_{(i-1) i}^{-}, \epsilon^{(i+1) i}, \beta_{i}^{-}, \epsilon^{(i-1) i} .
$$

The four-holed sphere $N$ filled by the Farey neighbors $\epsilon^{(i+1) i}, \beta_{i}^{ \pm}$and $\epsilon^{i(i-1)}, \beta_{i}^{ \pm}$ has holes corresponding to the $i$-th puncture and the curves $\beta$ and $\epsilon^{(i+1)(i-1)}$; see the right side of Figure 14. Only $\epsilon^{(i+1)(i-1)}$ intersects $\beta_{(i-1) i}^{ \pm}, \beta_{i(i+1)}^{ \pm}, \epsilon^{(i-1) i}, \epsilon^{i(i+1)}$ nontrivially, as required for Lemma 2.4.
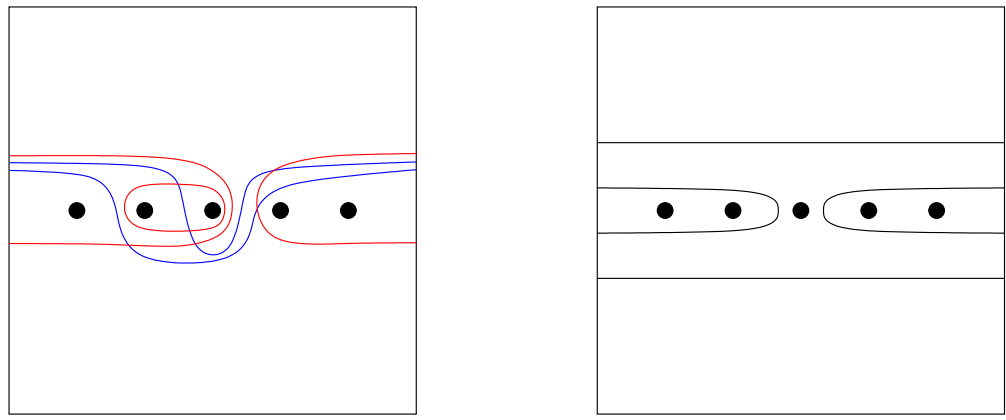

Figure 14. The curves $\beta_{23}^{-}, \epsilon^{43}, \beta_{3}^{-}, \epsilon^{23}$ on the left. The fourholed sphere filled by $\epsilon^{43}$ and $\beta_{3}^{ \pm}$on the right. 


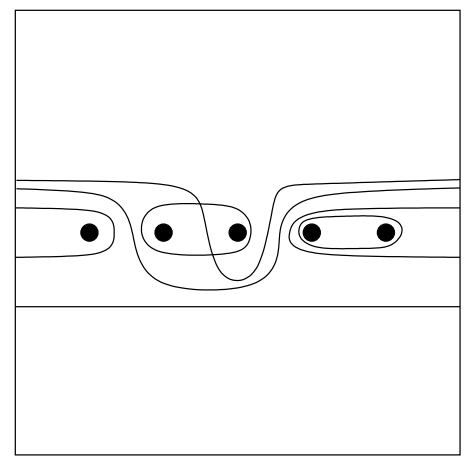

Figure 15. We use $\left\{\beta, \beta_{23}^{-}, \epsilon^{45}, \epsilon^{41}\right\}$ to detect $i\left(\beta_{3}^{-}, \epsilon^{23}\right) \neq 0$.

To see that all the intersections are $\mathfrak{X}_{1}$-detectable, we need only exhibit the necessary curves in $\mathfrak{X}_{1}$ determining a pants decomposition of $S-N$. See Figure 15 for the curves necessary to detect $i\left(\beta_{i}^{-}, \epsilon^{(i-1) i}\right) \neq 0$. We leave the other cases to the reader.

We are now in a position to prove Proposition 6.2.

Proof of Proposition 6.2. Let $\phi: \mathfrak{X}_{1} \rightarrow \mathscr{C}\left(S_{1, n}\right)$ be a locally injective simplicial map. By Theorem 6.1, there exists $f \in \operatorname{Mod}^{ \pm}\left(S_{1, n}\right)$ such that $\left.f\right|_{\mathfrak{X}}=\left.\phi\right|_{\mathfrak{X}}$, unique up to precomposing with $r$. In fact, after precomposing $\phi$ with $f^{-1}$ we may as well assume that $\left.\phi\right|_{\mathfrak{X}}$ is the identity.

According to Lemma 6.3, for all $i, \phi\left(\epsilon^{(i+1) i}\right)=\epsilon^{(i+1) i}$ and $\phi\left(\epsilon^{i(i-1)}\right)=\epsilon^{i(i-1)}$ are the unique Farey neighbors of $\phi\left(\beta_{i}^{-}\right)$and $\phi\left(\beta_{i}^{+}\right)$(with indices taken modulo $n$ ). Consequently, $\phi\left(\left\{\beta_{i}^{ \pm}\right\}\right)=\left\{\beta_{i}^{ \pm}\right\}$for all $i$. Notice that $i\left(\beta_{i}^{+}, \beta_{j}^{-}\right)=0$ for all $i, j$, while $i\left(\beta_{i}^{+}, \beta_{j}^{+}\right)=i\left(\beta_{i}^{-}, \beta_{j}^{-}\right)=2$ for all $i, j$. It follows that if $\phi\left(\beta_{i}^{-}\right)=\beta_{i}^{+}$for some $i$, then this is true for all $i$. Composing with $r$ if necessary, we deduce that $\phi\left(\beta_{i}^{ \pm}\right)=\beta_{i}^{ \pm}$for all $i$. All that remains is to see that $\phi\left(\beta_{i(i+1)}^{ \pm}\right)=\beta_{i(i+1)}^{ \pm}$for all $i$.

To prove this we need only show that

$$
\beta_{i(i+1)}^{ \pm} \in\left(\mathfrak{X} \cup\left\{\beta_{j}^{ \pm} \mid 1 \leq j \leq n\right\}\right)^{\prime},
$$

and then we can apply Proposition 3.5. First note that if $n=3$, then $\beta_{i(i+1)}^{ \pm}=\beta_{i+2}^{\mp}$, so there is nothing to prove in this case. In general, one readily checks that $\beta_{i(i+1)}^{+}$ is uniquely determined by the almost filling set

$$
\left\{\beta, \beta_{1}^{-}, \beta_{2}^{-}, \ldots, \beta_{n}^{-}\right\} \backslash\left\{\beta_{i}^{-}, \beta_{i+1}^{-}\right\} .
$$

This completes the proof.

Let $\mathfrak{O}_{P}=\left\{\epsilon^{i(i+1)}\right\}_{i=1}^{n}$, counting indices modulo $n$. For $n \geq 5$, this is a closed string of twistable Farey neighbors in $\mathfrak{X}_{1}$, and we could appeal to Proposition 3.12 
to add the half-twists about curves in $\mathfrak{O}_{P}$ in this case. However, we can provide a single argument for all $n \geq 3$.

Lemma 6.4. For all $\epsilon, \epsilon^{\prime} \in \mathfrak{O}_{P}, H_{\epsilon}^{ \pm 1}\left(\epsilon^{\prime}\right) \in \mathfrak{X}^{\prime}$. Consequently, $H_{\epsilon}^{ \pm 1}\left(\mathfrak{X}_{1}^{\prime}\right) \cup \mathfrak{X}_{1}^{\prime}$ is rigid.

Proof. We start with the proof of the first statement. If $i\left(\epsilon, \epsilon^{\prime}\right)=0$, then there is nothing to prove. Otherwise, up to a homeomorphism we may assume that $\epsilon=\epsilon^{i(i+1)}$ and $\epsilon^{\prime}=\epsilon^{(i+1)(i+2)}$. Then we note that $H_{\epsilon^{i(i+1)}}\left(\epsilon^{(i+1)(i+2)}\right)$ is the curve uniquely determined by the almost filling set of curves

$$
\left\{\beta_{i+1}^{+}\right\} \cup\left\{\alpha_{1}, \ldots, \alpha_{n}\right\} \backslash\left\{\alpha_{i+1}, \alpha_{i+2}\right\},
$$

completing the proof of the first statement.

For the second statement, we note that $H_{\epsilon^{i(i+1)}}\left(\mathfrak{X}_{1}^{\prime}\right) \cap \mathfrak{X}_{1}^{\prime}$ contains the weakly rigid set $\mathfrak{O}_{P} \cup\left\{\beta_{i+2}^{+}\right\}$, for example. Therefore, since $\mathfrak{X}_{1}$ is rigid by Proposition 6.2, so is $\mathfrak{X}_{1}^{\prime}$ by Proposition 3.5, and hence by Lemma 3.7 it follows that $H_{\epsilon^{i(i+1)}}\left(\mathfrak{X}_{1}^{\prime}\right) \cup \mathfrak{X}_{1}^{\prime}$ is rigid, as required. A similar argument proves the statement for $H_{\epsilon^{i(i+1)}}^{-1}$.

We also need to consider Dehn twists in $\alpha_{i}$ and $\beta$. To deal with these, we first define $\mathfrak{X}_{2}=\mathfrak{X}_{1}^{\prime} \cup H_{\mathfrak{O}_{P}}\left(\mathfrak{X}_{1}^{\prime}\right)$, where $H_{\mathfrak{O}_{P}}\left(\mathfrak{X}_{1}^{\prime}\right)$ is the union of $H_{\epsilon}^{ \pm 1}\left(\mathfrak{X}_{1}^{\prime}\right)$ over all $\epsilon \in \mathfrak{O}_{P}$. By Lemma 6.4, $\mathfrak{X}_{2}$ is rigid.

Lemma 6.5. For all $i=1, \ldots, n$, we have $T_{\alpha_{i}}^{ \pm 1}(\beta)=T_{\beta}^{\mp 1}\left(\alpha_{i}\right) \in \mathfrak{X}_{1}^{2} \subset \mathfrak{X}_{2}^{2}$. Consequently, $T_{\alpha_{i}}^{ \pm 1}\left(\mathfrak{X}_{2}^{2}\right) \cup \mathfrak{X}_{2}^{2}$ and $T_{\beta}^{ \pm 1}\left(\mathfrak{X}_{2}^{2}\right) \cup \mathfrak{X}_{2}^{2}$ are rigid.

Proof. As in previous arguments, we exhibit a series of pictures that will yield the desired result; see Figure 16. It is straightforward to modify such pictures to treat the case of an arbitrary $n \geq 3$. The top left picture shows an almost filling set in $\mathfrak{X}_{1}$ that uniquely determines a curve in $\mathfrak{X}_{1}^{\prime}$ on the top right. Then the lower left is an almost filling set in $\mathfrak{X}_{1}^{\prime}$ that uniquely determines the curve in $\left(\mathfrak{X}_{1}^{\prime}\right)^{\prime}=\mathfrak{X}_{1}^{2}$. This curve is precisely $T_{\beta}\left(\alpha_{i}\right)=T_{\alpha_{i}}^{-1}(\beta)$. Similarly, $T_{\alpha_{i}}(\beta)=T_{\beta}^{-1}\left(\alpha_{i}\right) \in \mathfrak{X}_{1}^{2}$.

Finally, we easily observe that $\mathfrak{X}_{2}^{2} \cap T_{\alpha_{i}}\left(\mathfrak{X}_{2}^{2}\right)$ is weakly rigid, as it contains $\mathfrak{C} \cup H_{\epsilon^{(i-1) i}}\left(\alpha_{i-1}\right)$, which is weakly rigid. Appealing to Lemma 3.7, it follows that $\mathfrak{X}_{2}^{2} \cup T_{\alpha_{i}}\left(\mathfrak{X}_{2}^{2}\right)$ is rigid. The other cases follow similarly.

Finally, we prove our main result for surfaces of genus 1 .

Proof of Theorem 1.1 for $S=S_{1, n}$. Since $\mathfrak{X}_{2}$ is rigid, by Propositions 3.5, the set $Y=\mathfrak{X}_{2}^{2}$ is rigid. Moreover, $\operatorname{Mod}\left(S_{1, n}\right) \cdot Y=\mathscr{C}\left(S_{1, n}\right)$, by inspection. A generating set for $\operatorname{Mod}\left(S_{1, n}\right)$ is given by the Dehn twists $f_{\alpha}$ about the elements $\alpha \in \mathfrak{C}$ and the half-twists $f_{\epsilon}$ about the elements $\epsilon \in A=\left\{\epsilon^{i(i+1)}\right\}$ (see Section 4.4 of [Farb and Margalit 2012], for instance). Let $G=\mathfrak{C} \cup A$ and note that, for each $\alpha \in G$, the set $Y \cup f_{\alpha} Y$ is rigid. Therefore, we may apply Proposition 3.13 to obtain the desired exhaustion of $\mathscr{C}\left(S_{1, n}\right)$ by finite rigid sets. 

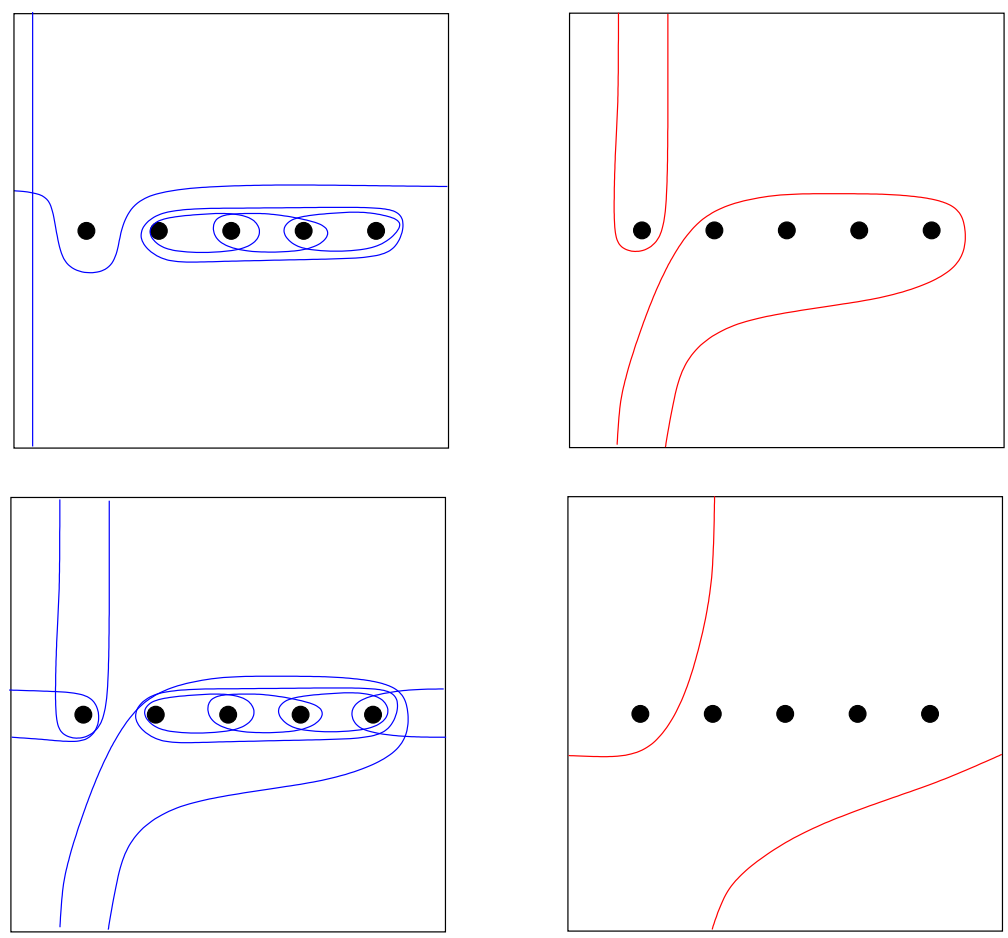

Figure 16. Illustrating $T_{\beta}\left(\alpha_{2}\right) \in \mathfrak{X}_{2}^{2}$ on $S_{1,5}$.

\section{Acknowledgements}

We thank Brian Bowditch for conversations and for his continued interest in this work. We also thank Juan Souto for conversations. Finally, we thank the referee for her/his comments. Parts of this paper were completed during the conference "Mapping class groups and Teichmüller Theory"; we would like to express our gratitude to Michah Sageev and the Technion for their hospitality and financial support.

\section{References}

[Aramayona and Leininger 2013] J. Aramayona and C. J. Leininger, "Finite rigid sets in curve complexes”, J. Topol. Anal. 5:2 (2013), 183-203. MR 3062946 Zbl 1277.57017

[Aramayona and Souto 2013] J. Aramayona and J. Souto, "A remark on homomorphisms from right-angled Artin groups to mapping class groups", C. R. Math. Acad. Sci. Paris 351:19-20 (2013), 713-717. MR 3125410 Zbl 1283.57005

[Birman et al. 2015] J. S. Birman, N. Broaddus, and W. W. Menasco, "Finite rigid sets and homologically nontrivial spheres in the curve complex of a surface", J. Topol. Anal. 7:1 (2015), 47-71. MR 3284389 Zbl 1308.57009 
[Broaddus 2012] N. Broaddus, "Homology of the curve complex and the Steinberg module of the mapping class group”, Duke Math. J. 161:10 (2012), 1943-1969. MR 2954621 Zbl 1250.57032

[Farb and Margalit 2012] B. Farb and D. Margalit, A primer on mapping class groups, Princeton Mathematical Series 49, Princeton University Press, 2012. MR 2012h:57032 Zbl 1245.57002

[Harer 1986] J. L. Harer, "The virtual cohomological dimension of the mapping class group of an orientable surface", Invent. Math. 84:1 (1986), 157-176. MR 87c:32030 Zbl 0592.57009

[Ivanov 1997] N. V. Ivanov, "Automorphism of complexes of curves and of Teichmüller spaces", Int. Math. Res. Notices 1997:14 (1997), 651-666. MR 98j:57023 Zbl 0890.57018

[Kim and Koberda 2013] S.-H. Kim and T. Koberda, "Embedability between right-angled Artin groups”, Geom. Topol. 17:1 (2013), 493-530. MR 3039768 Zbl 1278.20049

[Kim and Koberda 2015] S.-H. Kim and T. Koberda, "Right-angled Artin groups and finite subgraphs of curve graphs", Osaka J. Math. (online publication May 2015).

[Korkmaz 1999] M. Korkmaz, "Automorphisms of complexes of curves on punctured spheres and on punctured tori”, Topology Appl. 95:2 (1999), 85-111. MR 2000d:57025 Zbl 0926.57012

[Luo 2000] F. Luo, "Automorphisms of the complex of curves", Topology 39:2 (2000), 283-298. MR 2000j:57045 Zbl 0951.32012

[Shackleton 2007] K. J. Shackleton, "Combinatorial rigidity in curve complexes and mapping class groups”, Pacific J. Math. 230:1 (2007), 217-232. MR 2008g:57018 Zbl 1165.57017

Received October 24, 2014. Revised September 11, 2015.

JAVIER ARAMAYONA

DEPARTAMENTO DE MATEMÁTICAS

UNIVERSIDAD AUTÓNOMA DE MADRID

CiUdAD UNIVERSITARIA DE CANTOBLANCO

28049 MADRID

SPAIN

aramayona@gmail.com

Christopher J. LeININGER

DEPARTMENT OF MATHEMATICS

UNIVERSITY OF ILLINOIS AT URBANA-CHAMPAIGN

1409 W. GREEN STREET

URBANA, IL 61801

UNITED STATES

c.j.leininger95@gmail.com 


\title{
PACIFIC JOURNAL OF MATHEMATICS
}

\author{
msp.org/pjm
}

Founded in 1951 by E. F. Beckenbach (1906-1982) and F. Wolf (1904-1989)

\section{EDITORS}

Don Blasius (Managing Editor)

Department of Mathematics

University of California

Los Angeles, CA 90095-1555

blasius@math.ucla.edu

\author{
Paul Balmer \\ Department of Mathematics \\ University of California \\ Los Angeles, CA 90095-1555 \\ balmer@math.ucla.edu \\ Robert Finn \\ Department of Mathematics \\ Stanford University \\ Stanford, CA 94305-2125 \\ finn@math.stanford.edu \\ Sorin Popa \\ Department of Mathematics \\ University of California \\ Los Angeles, CA 90095-1555 \\ popa@math.ucla.edu
}

\author{
Vyjayanthi Chari \\ Department of Mathematics \\ University of California \\ Riverside, CA 92521-0135 \\ chari@math.ucr.edu \\ Kefeng Liu \\ Department of Mathematics \\ University of California \\ Los Angeles, CA 90095-1555 \\ liu@math.ucla.edu \\ Jie Qing \\ Department of Mathematics \\ University of California \\ Santa Cruz, CA 95064 \\ qing@ cats.ucsc.edu
}

\section{PRODUCTION}

Silvio Levy, Scientific Editor, production@msp.org

\section{SUPPORTING INSTITUTIONS}

ACADEMIA SINICA, TAIPEI

CALIFORNIA INST. OF TECHNOLOGY

INST. DE MATEMÁTICA PURA E APLICADA

KEIO UNIVERSITY

MATH. SCIENCES RESEARCH INSTITUTE

NEW MEXICO STATE UNIV.

OREGON STATE UNIV.

\author{
STANFORD UNIVERSITY \\ UNIV. OF BRITISH COLUMBIA \\ UNIV. OF CALIFORNIA, BERKELEY \\ UNIV. OF CALIFORNIA, DAVIS \\ UNIV. OF CALIFORNIA, LOS ANGELES \\ UNIV. OF CALIFORNIA, RIVERSIDE \\ UNIV. OF CALIFORNIA, SAN DIEGO \\ UNIV. OF CALIF., SANTA BARBARA
}

\author{
Daryl Cooper \\ Department of Mathematics \\ University of California \\ Santa Barbara, CA 93106-3080 \\ cooper@math.ucsb.edu \\ Jiang-Hua Lu \\ Department of Mathematics \\ The University of Hong Kong \\ Pokfulam Rd., Hong Kong \\ jhlu@maths.hku.hk \\ Paul Yang \\ Department of Mathematics \\ Princeton University \\ Princeton NJ 08544-1000 \\ yang@math.princeton.edu
}

These supporting institutions contribute to the cost of publication of this Journal, but they are not owners or publishers and have no responsibility for its contents or policies.

See inside back cover or msp.org/pjm for submission instructions.

The subscription price for 2016 is US $\$ 440 /$ year for the electronic version, and $\$ 600 /$ year for print and electronic.

Subscriptions, requests for back issues and changes of subscriber address should be sent to Pacific Journal of Mathematics, P.O. Box 4163, Berkeley, CA 94704-0163, U.S.A. The Pacific Journal of Mathematics is indexed by Mathematical Reviews, Zentralblatt MATH, PASCAL CNRS Index, Referativnyi Zhurnal, Current Mathematical Publications and Web of Knowledge (Science Citation Index).

The Pacific Journal of Mathematics (ISSN 0030-8730) at the University of California, c/o Department of Mathematics, 798 Evans Hall \#3840, Berkeley, CA 94720-3840, is published twelve times a year. Periodical rate postage paid at Berkeley, CA 94704, and additional mailing offices. POSTMASTER: send address changes to Pacific Journal of Mathematics, P.O. Box 4163, Berkeley, CA 94704-0163.

PJM peer review and production are managed by EditFLOW ${ }^{\circledR}$ from Mathematical Sciences Publishers.

\section{PUBLISHED BY}

\section{mathematical sciences publishers \\ nonprofit scientific publishing}

http://msp.org/

(C) 2016 Mathematical Sciences Publishers 


\section{PACIFIC JOURNAL OF MATHEMATICS}

Volume $282 \quad$ No. $2 \quad$ June 2016

Exhausting curve complexes by finite rigid sets

JAVIER ARAMAYONA and CHRISTOPHER J. LEININGER

A variational characterization of flat spaces in dimension three

Giovanni Catino, Paolo Mastrolia and Dario D. Monticelli

Estimates of the gaps between consecutive eigenvalues of Laplacian

293

DAgUang Chen, TAO Zheng and HongCANG YANG

Liouville type theorems for the $p$-harmonic functions on certain manifolds

JINGYI CHEN and YUE WANG

Cartan-Fubini type rigidity of double covering morphisms of quadratic manifolds

\section{HOSUNG KIM}

On the uniform squeezing property of bounded convex domains in $\mathbb{C}^{n}$

KANG-TAE KIM and LIYOU ZHANG

Lefschetz pencils and finitely presented groups

RYOMA KOBAYASHI and NAOYUKI MONDEN

Knot homotopy in subspaces of the 3-sphere

YUYA KODA and MAKOTO OzAWA

On the relationship of continuity and boundary regularity in prescribed mean curvature Dirichlet problems

KIRK E. LANCASTER and JARON MELIN

Bridge spheres for the unknot are topologically minimal

JUNG HOON LEE

On the geometric construction of cohomology classes for cocompact discrete subgroups of $\mathrm{SL}_{n}(\mathbb{R})$ and $\mathrm{SL}_{n}(\mathbb{C})$

SUSANNE SCHIMPF

On Blaschke's conjecture

Xiaole Su, Hongwei Sun and Yusheng Wang

The role of the Jacobi identity in solving the Maurer-Cartan structure equation 